\title{
Extended Nucleation and Superfocusing in Colloidal Semiconductor Nanocrystal Synthesis
}

\author{
Supporting Information
}

P. Tim Prins, Federico Montanarella, Kim Dümbgen, Yolanda Justo, Johanna C. van der Bok, Stijn O.M. Hinterding, Jaco J. Geuchies, Jorick Maes, Kim De Nolf, Sander Deelen, Hans Meijer, Thomas Zinn, Andrei V. Petukhov, Freddy T. Rabouw, Celso De Mello Donega, Daniel Vanmaekelbergh and Zeger Hens

Debye Institute for Nanomaterials Science, Utrecht University, Utrecht, The Netherlands

P. Tim Prins, Federico Montanarella, Johanna C. van der Bok, Stijn O.M. Hinterding, Jaco J. Geuchies, Andrei V. Petukhov, Freddy T. Rabouw, Celso De Mello Donega, Daniel Vanmaekelbergh

Physics and Chemistry of Nanostructures, Ghent University, Gent, Belgium

Kim Dümbgen, Yolanda Justo, Jorick Maes, Kim De Nolf, Zeger Hens

Scientific Instrumentation, Faculty of Science, Utrecht University, Utrecht, The Netherlands Sander Deelen, Hans Meijer

ID02, ESRF, Grenoble, France

Thomas Zinn

Laboratory of Physical Chemistry, Eindhoven University of Technology, The Netherlands Andrei V. Petukhov 


\section{S.1 In-situ reaction analysis by SAXS, experimental methods}

\section{S.I.I Chemicals}

Tri-octylphospine oxide (TOPO, 90\%), octadecylamine (ODA, 90\%), oleic acid (OA, 90\%), 1octadecene (ODE, 90\%), cadmium acetate dihydrate $\left(\mathrm{Cd}(\mathrm{Ac})_{2} \cdot 2 \mathrm{H}_{2} \mathrm{O}, 98 \%\right)$, tri-octylphosphine (TOP, 90\%), ethanol (EtOH, 99.8\%), 1-butanol anhydrous (BuOH, 99.8\%), methanol (MeOH, anhydrous, 99,8\%), and toluene (anhydrous, 99.8\%) were all purchased from Sigma Aldrich and used as received. Se powder (Se, 200 mesh, 99.999\%) was purchased from Brunschwig Chemie.

\section{S.I.2 Synthesis of CdSe Nanocrystals}

The synthesis procedure for the formation of CdSe nanocrystals (NCs) was adapted from literature. ${ }^{1}$

Precursor synthesis. For the preparation of the Cd-precursor, $1.76 \mathrm{~g} \mathrm{Cd}(\mathrm{Ac})_{2} .2 \mathrm{H}_{2} \mathrm{O}, 8.76 \mathrm{~g} \mathrm{OA}$ and $61.74 \mathrm{~g}$ ODE were mixed and degassed in a three-neck flask at $120^{\circ} \mathrm{C}$ for three hours to form $\mathrm{Cd}(\mathrm{OA})_{2}$ with $0.1 \mathrm{M}$ concentration. For the preparation of the Se-precursor, $5.15 \mathrm{~g} \mathrm{Se}$ powder, $27.24 \mathrm{~g}$ TOP and $43.22 \mathrm{~g}$ ODE (previously degassed) were heated to $50^{\circ} \mathrm{C}$ and stirred under inert atmosphere in a three-neck flask until complete dissolution of the Se powder. ODA and TOPO were separately degassed at $150^{\circ} \mathrm{C}$ for two hours and then kept under inert atmosphere until use.

Nanocrystal synthesis. For the synthesis of CdSe nanocrystals, $0.81 \mathrm{~g}$ tri-octylphosphine oxide (TOPO), $2.33 \mathrm{~g}$ octadecyl amine (ODA), and $3.78 \mathrm{~g}$ of the Se-precursor (see Supplementary Information S1 for precursor preparation) were placed in a custom-made three-neck flask. The content of the flask was heated to $290{ }^{\circ} \mathrm{C}$ under nitrogen. At $290{ }^{\circ} \mathrm{C}, 3.56 \mathrm{~g}$ of the Cd-precursor was rapidly injected in the flask via the remotely-controlled liquid injector. The temperature, which had dropped to $230{ }^{\circ} \mathrm{C}$ after the injection, was set to $260{ }^{\circ} \mathrm{C}$. The mixture reached this temperature within 60 seconds, the solution was kept at this temperature for 120 minutes while stirring. During this time the temperature oscillated around $260{ }^{\circ} \mathrm{C}$ with an amplitude of $6.5^{\circ} \mathrm{C}$ and a period of 195 seconds. After 120 minutes, the heating was turned off and the reaction was let cool down to room temperature. At room temperature, the crude solution was collected and stored under inert atmosphere for further analysis.

Sample purification. The samples for ex situ TEM and optical absorption analysis were prepared by addition of a mixture of $\mathrm{MeOH}$ and $\mathrm{BuOH}(1: 3)$ to the crude solution and then centrifugation at $2500 \mathrm{rpm}$ for 10 minutes. After centrifugation the supernatant was discarded and the precipitate was redispersed in $6 \mathrm{~mL}$ of toluene. The resulting QD solution, red and clear, was then used for the above-mentioned ex situ TEM and optical absorption analysis.

\section{S.I.3 Ex-situ materials analysis}

Figure S1.1 summarizes the basic characteristics (morphology, linear optical properties) of the CdSe nanocrystals synthesized using the procedure described in S.1.2. 
a

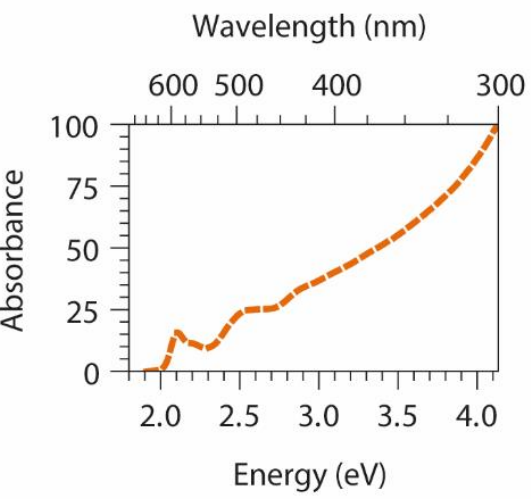

b

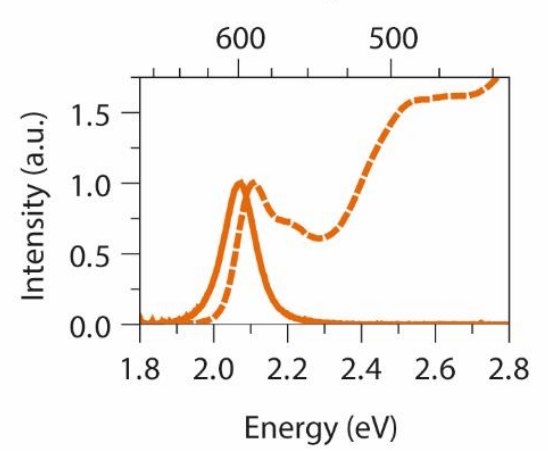

C

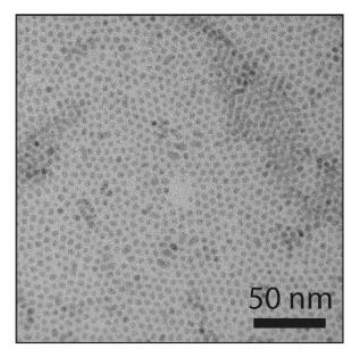

Figure S1.1. Optical and structural characterization of CdSe nanocrystals synthesized through hot injection. (a) Absorption spectrum of the purified synthesis products measured in a cuvette with a pathlength of $1 \mathrm{~cm}$. The first exciton transition is clearly visible at 2.10 $\mathrm{eV}$, according to a SAXS-based sizing curve, relating the first excitonic transition to radius, ${ }^{2}$ this corresponds to nanocrystals with a radius of $2.36 \mathrm{~nm}$, a close match with the final radius extracted from our in-situ SAXS measurements (see main text). The concentration nanocrystals can also be obtained, using the Lambert-Beer law and a molar extinction coefficient from literature $\left(3.3 \mu \mathrm{M}^{-1} \mathrm{~cm}^{-1}\right.$ at $300 \mathrm{~nm}$ for CdSe nanocrystals with a radius of $2.36 \mathrm{~nm}$ dispersed in toluene ${ }^{3}$ ) we get $30.6 \mu \mathrm{M}$. Considering the $25 \%$ thermal expansion of the solvent (see also section S.2.2.), the concentration at $260^{\circ} \mathrm{C}$ would be about $24 \mu \mathrm{M}$, in good agreement with the final concentration obtained from our in-situ SAXS measurements (see main text). (b) Normalized absorption and emission spectra of the CdSe nanocrystals product. The emission peaks at $2.27 \mathrm{eV}$ and has a full-width-half-maximum of $105 \mathrm{meV}$. (c) TEM image of the products of the synthesis. The nanocrystals have a quasi-spherical shape with a radius of $2.3 \pm 0.3 \mathrm{~nm}$.

\section{S.I.4 Setup for in-situ SAXS reaction monitoring}

Reaction flask. The synthesis was performed in a custom-made three-neck flask equipped with an indentation (Figure S1.2). The design of the flask was adapted from literature for our purposes, ${ }^{4}$ and it had the advantage of having the same design and structure of the regular threeneck flasks used for the synthesis of colloidal nanocrystals in the chemistry lab, while allowing to probe the sample with X-rays. The flask was carefully designed to allow proper mixing of the reagents, via the presence of a stirring bean at the bottom of it and regardless of the presence of the indentation. The distance between the inner window of the indentation and the outer window of the flask was $4 \mathrm{~mm}$, and it was designed to allow for the transmission of X-rays without complete absorption from the sample environment. The Duran ${ }^{\circledR}$ borosilicate glass 3.3 windows from Schott had a thickness of $0.7 \mathrm{~mm}$ and a diameter of $8 \mathrm{~mm}$. In order to perform the experiment, the flask was filled with the reagents and connected to the nitrogen supply, thus ensuring that the reaction would be performed under inert atmosphere. The flask was then wrapped with a heating ribbon connected to a thermocouple that probed the temperature of the reagents, thus allowing to reach the temperatures at which the synthesis was performed (260$\left.290^{\circ} \mathrm{C}\right)$. 

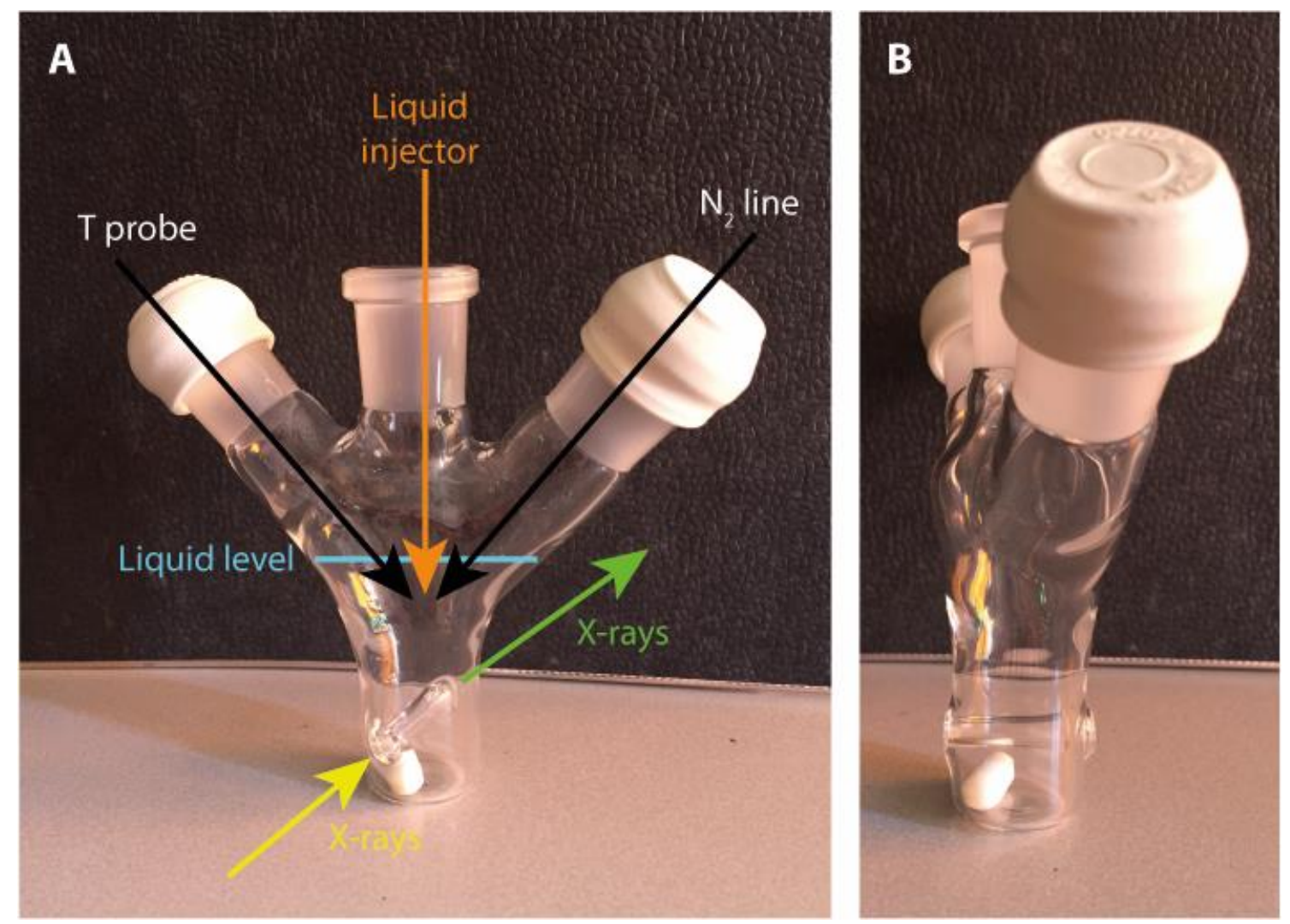

Figure S1.2. Custom-made three-neck flask. (a) Front and (b) side views of the custom-made three-neck flask used for the SAXS experiments. The flask was equipped with an indentation in its lower part, thus enabling the probing of a small part of the sample during the synthesis. The necks of the three-neck flask were used to insert a temperature probe, to connect the flask to a nitrogen line and to inject the precursors at high temperature.

The flask was then positioned inside a protective aluminum box with holes on both sides, at the same height as the indentation, and wide enough to allow the collection of SAXS and WAXS signals (Figure S1.3a-c). The box had the double purpose of securing the flask and containing potential spillages. The box constituted the upper part of a compact setup which included a stirring plate, in the middle, and an aluminum optical breadboard, at the bottom; this design was characterized by optimal compactness and user-friendliness. In order to perform the hot injection, the setup was equipped with a remotely-controlled liquid injector which was attached to the lower optical breadboard and positioned above the flask (Figure S1.3d). The injector was composed of two pistons, which were actuated by compressed air and that could be activated remotely independently. The pistons pushed on two different syringes which contained the reagents to be injected at high temperature and that were kept in place by rubber bands. 

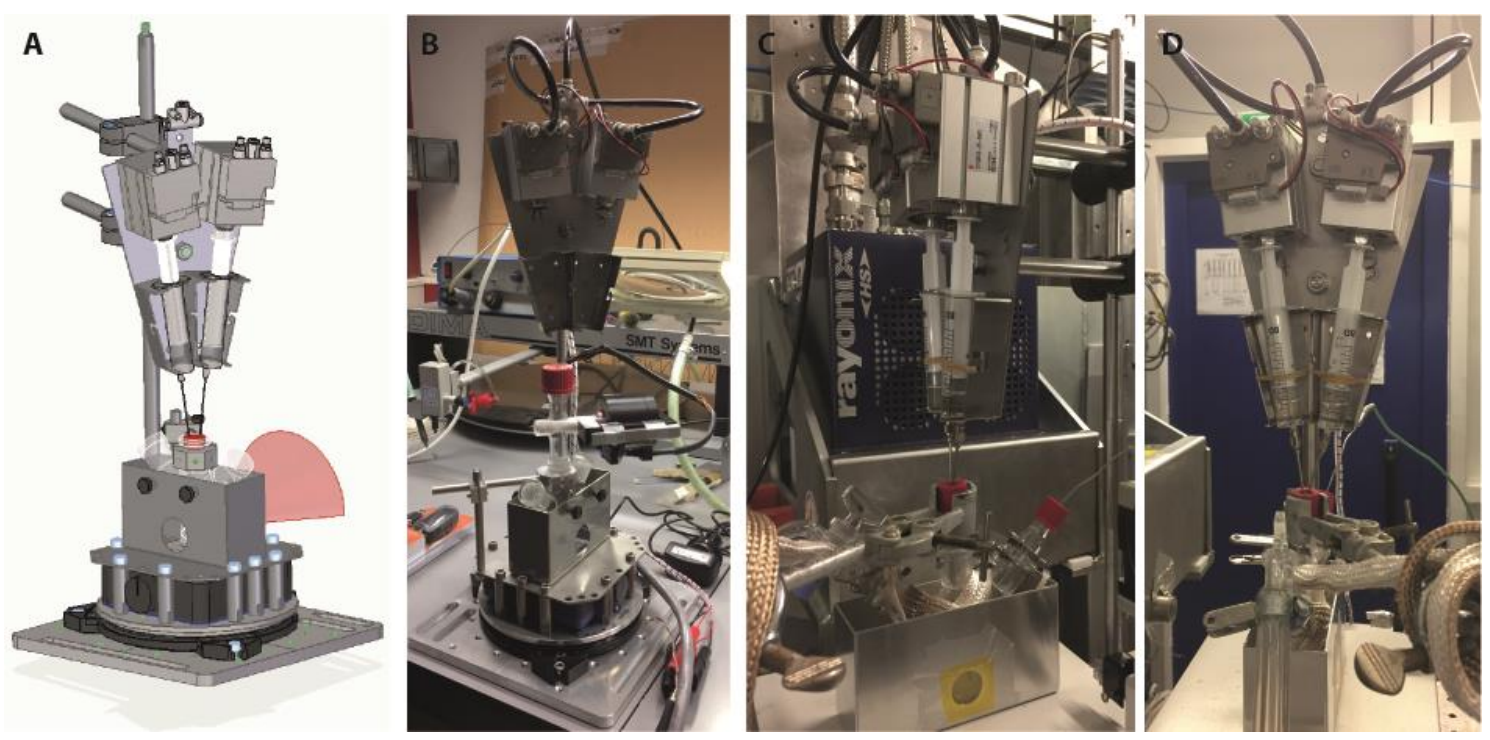

Figure S1.3. Custom-made setup for X-ray scattering experiments. (a) Scheme of the setup for X-ray scattering experiments. The custom-made flask was positioned inside a protective aluminum box with two holes at the same height as the indentation, so to allow collection of the SAXS/WAXS signal (red cone). The box was welded to a structure composed of a stirring plate and an aluminum optical breadboard. Above the flask, and connected to it through two metallic needles, were two syringes containing the reagents for the hot injection. The syringes were pressed by two pistons which could be activated remotely. (b) Digital photo of the setup in the workshop during manufacturing. (c) Digital photo of the setup, ready for an experiment, in the experimental hutch of ID02 at ESRF. (d) Digital photo of the custom-made liquid injector.

SAXS/WAXS measurements. The SAXS/WAXS experiments were performed on the ID02 beamline of the ESRF (European Synchrotron Radiation Facility) at an energy of $18 \mathrm{keV}( \pm 2$ $\mathrm{eV}$ ) with Rayonix MC-170HS detector and a sample-to-detector distance of 1.0 meter. This distance allowed us to probe a scattering vector $\boldsymbol{q}$ range between $0.1 \mathrm{~nm}^{-1}$ and $5 \mathrm{~nm}^{-1}$. The $\boldsymbol{q}$ range and the scattering intensity were calibrated as described in Ref. ${ }^{5}$. A detailed description of the beamline ${ }^{5}$ and the data treatments ${ }^{6}$ can be found in literature. Due to the fast kinetics of the reaction in the early stages of the synthesis, and due to the limited storage memory, the patterns were recorded with increasing time in between the patterns. The time in between the measurements followed the formula: $t_{i}+t_{w} 1.015^{n-1}$, where $t_{i}$ is the integration time $(75 \mathrm{~ms})$, $t_{w}$ the initial waiting/dead time $(22 \mathrm{~ms}$, the shortest time possible for the detector with $8 \times 8$ pixel binning), and $n$ the number of the frame with a maximum of 575 . This resulted in a temporal resolution of $100 \mathrm{~ms}$ at the beginning of the experiment and about 100 seconds at the end of the experiment.

\section{S.I.5 Ex-situ reaction development by quantitative aliquots}

As a benchmark of the in-situ SAXS study, we carried out the same synthesis of CdSe nanocrystals as described in S.1.2 in a standard lab set-up similar on monitored the reaction development by means of the UV-Vis absorption spectrum of quantitative reaction aliquots. More details on the quantitative analysis of absorption spectra are provided in section S.3. 

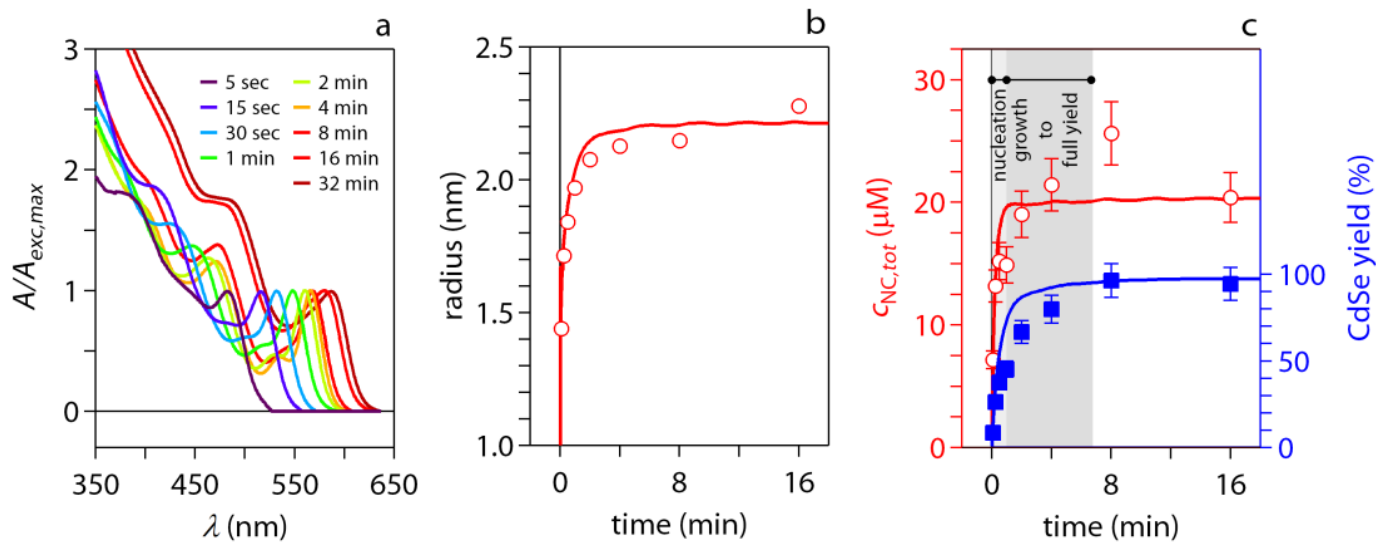

Figure S1.2. (a) Absorbance spectra of reaction aliquot taken as indicated after the start of a CdSe synthesis following the method used for the in-situ SAXS study. (b) (markers) Nanocrystal radius as obtained from the central wavelength of the first exciton absorption, using a SAXS-based calibration curve. (full line) Development of the nanocrystal radius as obtained from the in-situ SAXS analysis - the same data have been represented in Figure 1. (c) (blue squares) yield of $\mathrm{CdSe}$ and (red circles) nanocrystal concentration as obtained from the aliquot analysis. In both cases, the full lines represent the data obtained from the in-situ SAXS analysis. Note that we multiplied the in-situ SAXS based concentration by 0.8 to account for the slight underestimation of the reaction yield in the aliquot analysis.

Figure S1.2 depicts the different absorption spectra, together with the time development of the estimated average radius, the nanocrystal concentration and the CdSe yield. As can be seen in Figure S1.2a, the first exciton feature shifts to longer wavelengths and becomes more pronounced with increasing reaction time, an evolution reflecting nanocrystal growth and size focusing. Comparing the data estimated from the absorption spectra and the in-situ SAXS analysis, one sees that the aliquot study has a somewhat smaller reaction yield between 1 and 4 minutes, possibly related to difficulty to impose exactly the same temperature program in both cases. While this results in a slight underestimation of the nanocrystal concentration, we can conclude that both analysis methods lead to a very similar picture of the reaction development that includes in both cases a gradual buildup of the nanocrystal concentration. Therefore, we can rule out an impact of the x-ray irradiation on the CdSe formation, and we can use aliquot studies as a rapid and easily accessible method to analyze the reaction development. 


\section{S.2 Analysis of SAXS patterns}

\section{S.2.I SAXS patterns}

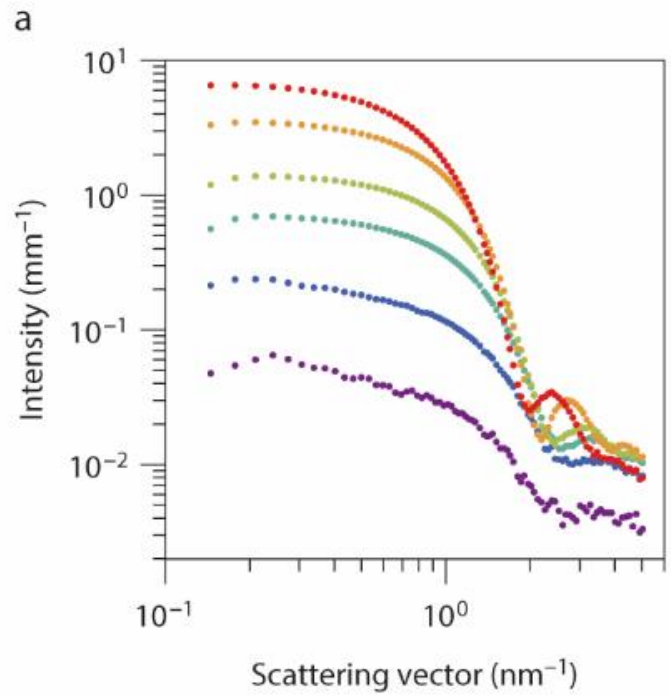

b

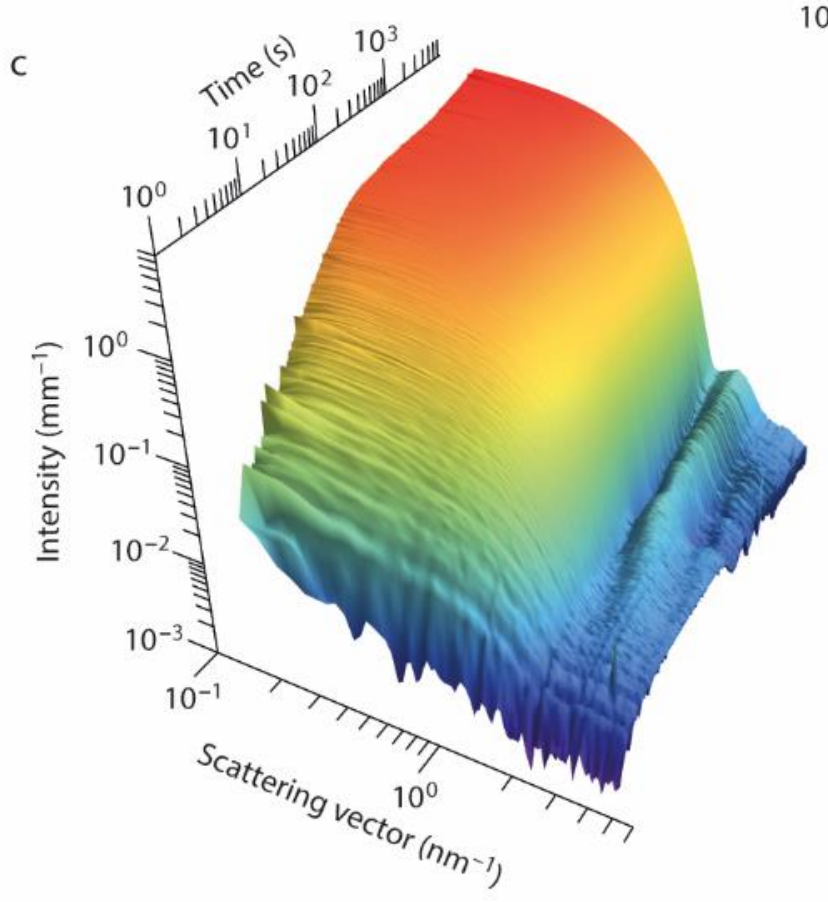

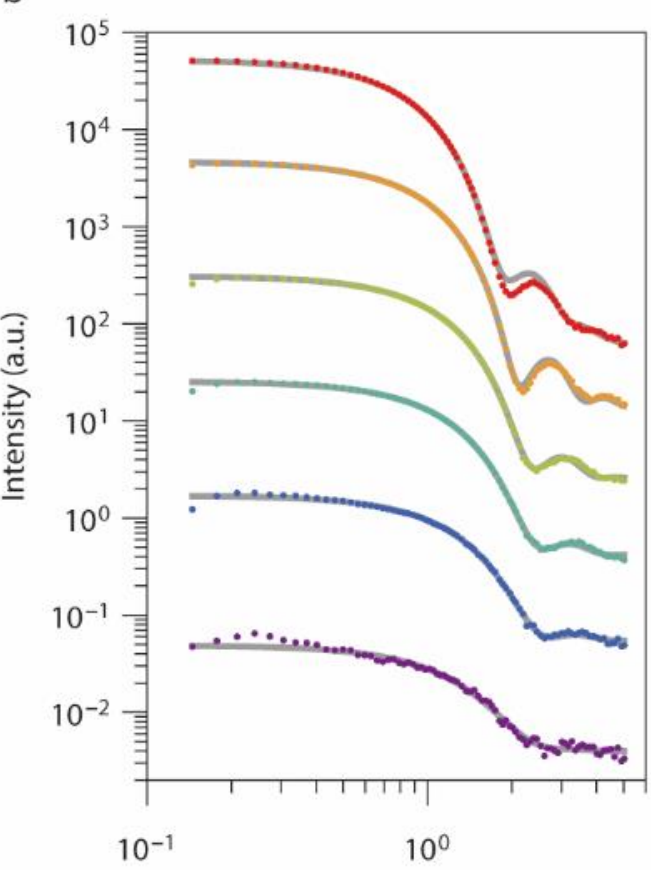

Scattering vector $\left(\mathrm{nm}^{-1}\right)$

Figure S2.1. 1D scattering patterns extracted from the 2D scattering patterns and their corresponding fits. From purple to red are respectively corresponding to the times $1.8 \mathrm{~s}, 5.3$ s, $11 \mathrm{~s}, 22 \mathrm{~s}, 76 \mathrm{~s}$, and $78 \mathrm{~min}$ after the hot injection. (a) The 1D scattering patterns with absolute intensity and background subtracted at the selected times. (b) The scattering patterns from panel a but now shifted for clarity (with $6^{n-1}, n$ the number of the scattering pattern) and their corresponding fits. (c) All 1D scattering patterns collected during the hot injection synthesis with background subtracted and absolute intensities.

For isotropic samples, the scattering intensity does not depend on the azimuthal angle of the detector. For this reason, we only discuss the data analysis of the one-dimensional (1D) 
scattering patterns obtained by azimuthal integration of the $2 \mathrm{D}$ scattering patterns, performed by a procedure specific for the used beamline, ID02 at the ESRF, as described by Ref. ${ }^{6}$. Examples of the 1D scattering patterns are shown in Figure S2.1a for different times after the hot injection. From these scattering patterns the background (scattering pattern at time $1 \mathrm{~s}$ ) is already subtracted. Absolute intensities were obtained by correcting for the pathlength, $4 \mathrm{~mm}$, calibrated by measuring the scattering intensity of water (absolute scattering cross section 1.65 $\left.10^{-3} \mathrm{~mm}^{-1}\right){ }^{7}$ A comprehensive overview of common protocols for the handling of scattering patterns on semiconducting nanocrystals is described by Ref. ${ }^{2}$. Figure S2.1.c shows the scattering patterns with background subtracted and absolute intensities collected during the hot injection synthesis as used for further data analysis.

\section{S.2.2 Data analysis}

\section{a}

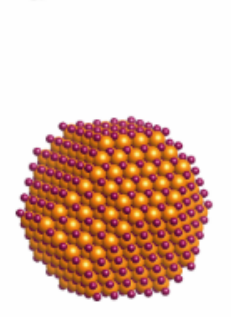

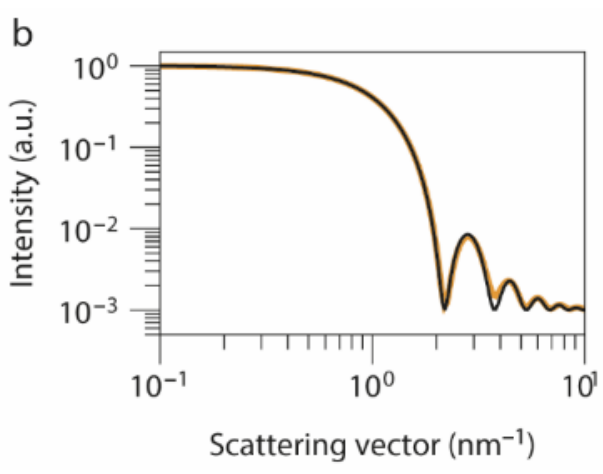

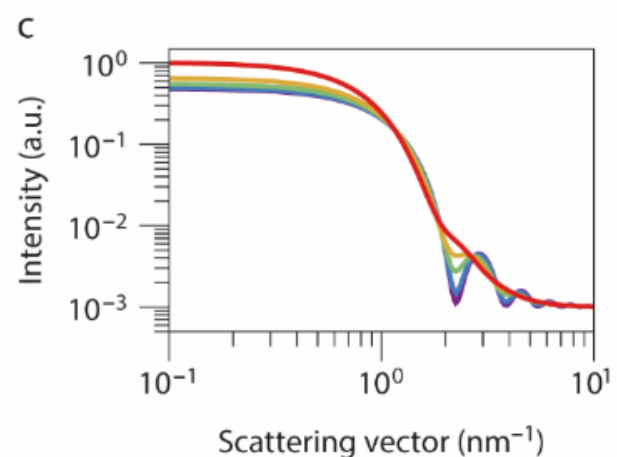

Figure S2.2. SAXS modeling and fitting. (a) Schematic representation of a faceted CdSe nanocrystal, cadmium atoms are depicted red and the selenium atoms orange (produced with Vesta). ${ }^{8}$ The nanocrystal has a zinc blende crystal structure with six [100] and eight [111] facets at the surface, its shape is that of a truncated cube. This representation is closer to the real shape of the nanocrystals prepared in our work than the perfectly spherical shape used to fit the SAXS data. (b) Scattering curve of the nanocrystal shown in panel a, taking into account all the individual atoms, i.e. the exact shape represented in panel (a) (orange curve), and all orientations. The black curve is the theoretical scattering curve of a perfect sphere with radius $2.06 \mathrm{~nm}$. It is clear that approximating the shape of the nanocrystals to a perfect sphere does not induce significant differences in the scattering curve with respect to the more realistic multifaceted and isotropic shape depicted in panel a. (c) Theoretical scattering patterns of an ensemble of nanocrystals with average radius of $2 \mathrm{~nm}$ and polydispersities of $2.5 \%, 5 \%, 10 \%, 15 \%, 25 \%$ for respectively purple to red.

Assumptions. Since the reaction mixture is a diluted mixture of nanocrystals with a volume fraction $f \sim 7 \cdot 10^{-4}$ at the end of the synthesis, we analysed the SAXS patterns assuming that there is no interaction between the nanocrystals, i.e., the structure factor is equal to 1. Ex situ transmission electron microscopy images show that the nanocrystals are quasi-spherical (Figure S1.1a). In reality the nanocrystals are faceted as shown in Figure S2.2a. However, the difference in SAXS between a perfect sphere and a multifaceted and isotropic nanocrystal is minimal (Figure S2.2b). Hence, we use the assumption that the nanocrystals are spherical with a radius $R$, which follow a Gaussian distribution.

Model scattering pattern. The scattering pattern of a mixture of nanocrystals with an isotropic form factor $P_{\text {sphere }}$ and a Gaussian distribution of radii reads: ${ }^{9}$ 


$$
I(q)=c_{\mathrm{NC}, \text { tot }} N_{\mathrm{A}} P_{\text {sphere }}(q)
$$

Here, $c_{\mathrm{NC}, \text { tot }}$ is the molar concentration of nanocrystals, $N_{\mathrm{A}}$ the Avogadro constant, and the isotropic form factor $P_{\text {sphere }}$ for a spherical object is given by:

$$
P_{\text {sphere }}(q)=36 \pi \Delta \rho^{2} V_{\text {sphere }}^{2} \frac{(\sin q R-q R \cos q R)^{2}}{(q R)^{6}}
$$

The average form factor for spheres with a Gaussian distribution of radii $R$ is then obtained as:

$$
\begin{aligned}
& \left\langle P_{\text {sphere }}(q)\right\rangle_{R} \\
& =36 \pi \Delta \rho^{2} \frac{1}{\sqrt{2 \pi} \sigma_{R}} \int_{0}^{\infty} e^{-\frac{1}{2}\left(\frac{R-R_{0}}{\sigma_{R}}\right)^{2}} V_{\text {sphere }}^{2} \frac{(\sin q R-q R \cos q R)^{2}}{(q R)^{6}} d R
\end{aligned}
$$

Here, $R_{0}$ is the mean radius and $\sigma_{R}$ the standard deviation. Figure $\mathrm{S} 2.2 \mathrm{c}$ shows the influence of the standard deviation on the theoretical scattering pattern. The increase in intensity at small scattering vector values is due to the increase in the average volume of the nanocrystals.

$\Delta \rho$ is the scattering length density contrast, given by:

$$
\Delta \rho=\rho_{\text {CdSe }}-\rho_{\text {reaction mixture }}
$$

According to the scattering length density calculator provided by the National Institute of Standards and Technology (NIST, NIST, https://www.ncnr.nist.gov/resources/activation/), we have $\rho_{\text {CdSe }}=40.710^{-6} \AA^{-2}$ and $\rho_{\text {reaction mixture }}=5.510^{-6} \AA^{-2}$ at $18 \mathrm{keV}$ and $260{ }^{\circ} \mathrm{C}$. Note: At room temperature the density of the reaction mixture is $0.8 \mathrm{~g} / \mathrm{cm}^{3}$. However, for organic materials the thermal expansion is significant, and based on Refs ${ }^{10-11}$ we estimated a thermal expansion of $25 \%$ from room temperature to $260^{\circ} \mathrm{C}$. For that reason a density of 0.6 $\mathrm{g} / \mathrm{cm}^{3}$ was used to calculated $\rho_{\text {reaction mixture }}$.

Fitting procedure. Fitting of the experimental data was done following a weighted least squares fitting procedure in a custom script. It was weighted with $1 / \sigma^{2}, \sigma$ the standard deviation on the measured intensity values provided in the processed data from the beamline, ID02 at the ESRF. The standard deviation is influenced by the number of counts on a pixel and the number of pixels corresponding to a certain $\mathrm{q}$ bin. ${ }^{12}$ Propagation of error was considered when rebinning the data and subtracting the background. Equation S2.1 with S2.3 were used as model, integrated by summation with steps of $0.02 \mathrm{~nm}$, plus an additional constant background. From this fitting procedure we extracted the molar concentration, average radius, standard deviation, and background as function of time as shown in the main text Figure 1b-c. Examples of the obtained fits are shown for selected times in Figure S2.1b.

\section{S.2.3 Reaction yield calculation}

From the fits to the SAXS scattering pattern, we obtained the molar concentration and the average radius of the CdSe nanocrystals in the reaction mixture. To calculate from these data the reaction yield, we first determined the average number $n_{C d^{2+}}$ of $\mathrm{Cd}^{2+}$ cations in a single nanocrystal from the ratio between the average nanocrystal volume $\left\langle V_{\text {sphere }}\right\rangle_{R}$ and the volume of the CdSe zinc blende unit cell ( $a$ : lattice parameter): 


$$
n_{C d^{2+}}=4 \frac{\left\langle V_{\text {sphere }}\right\rangle_{R}}{a^{3}}
$$

The reaction yield $Y$ is then obtained from the ratio between the total amount of $\mathrm{Cd}^{2+}$ cations incorporated in the nanocrystals and the amount of $\mathrm{Cd}^{2+}$ cations initially present in the reaction mixture:

$$
Y=c_{\mathrm{NC}, t o t} \frac{n_{C d^{2+}}}{c_{\mathrm{Cd}, 0}}
$$

Here, $c_{\mathrm{NC} \text {,tot }}$ is the molar nanocrystal concentration as deduced from the SAXS analysis, and $c_{\mathrm{Cd}, 0}$ is the initial molar concentration of the Cd-precursor. 


\section{S.3 Aliquot-analysis of reaction development}

\section{S.3.I CdSe nanocrystal synthesis}

Synthesis method. CdSe nanocrystals were synthesized as described in ref. ${ }^{13}$. In brief, a $10 \mathrm{~mL}$ mixture of $0.2 \mathrm{mmol}$ cadmium stearate, $0.4 \mathrm{mmol}$ stearic acid, and $1.6 \mathrm{mmol}$ hexadecylamine (HDA) in octadecene was loaded in a $25 \mathrm{~mL}$ three-neck flask. The reaction mixture was degassed for 30-60 min at room temperature and $60 \mathrm{~min}$ at $100{ }^{\circ} \mathrm{C}$ under a nitrogen flow. Afterwards, the temperature was raised to an injection temperature of $245^{\circ} \mathrm{C}$ and $2 \mathrm{~mL}$ of a solution of $0.5 \mathrm{mmol} \mathrm{Se}$ in tri-octylphosphine (TOP-Se) was injected. After injection, the reaction temperature was stabilized at $230{ }^{\circ} \mathrm{C}$. The reaction was monitored by means of successive aliquots and finally quenched by cooling with a water bath.

Aliquots. During the reaction, aliquots were taken and quenched in a 1:5 mixture of oleic acid and toluene with a known weight. After determining the mass of the aliquot by weighting, the nanocrystals were precipitated with a 1:1 mixture of methanol and 2-propanol. The precipitate was redissolved in toluene, precipitated a second time with methanol, and again redissolved in toluene. As demonstrated in the literature, this synthesis yields quasi-spherical $\mathrm{CdSe}$ nanocrystals with a zinc blende structure. ${ }^{13-14}$

Aliquot analysis. To determine the amount of $\mathrm{CdSe}$ in a given aliquot, we used an average determined by combining the absorbance at 340, 320 and $300 \mathrm{~nm}$ with published respective intrinsic absorption coefficients at these wavelengths and we assumed a fixed 1.2:1 Cd:Se ratio in the nanocrystals. ${ }^{3}$ The radius was determined from the wavelength where the exciton absorbance peaks using a published, SAXS-based calibration curve. ${ }^{2}$ Both numbers were combined to determine the CdSe nanocrystal concentration.

Data analysis - CdSe formation. It has been shown in the literature that the time development of the amount of CdSe $\left(n_{\mathrm{CdSe}}(t)\right)$ in the reaction is in line with a rate-determining monomer generation rate that is first order in the cadmium and first order in the selenium concentration: ${ }^{13}$

$$
n_{\mathrm{CdSe}}(t)=n_{\mathrm{Cd}, 0}\left(1-\frac{(\beta-1) e^{-k_{2}(\beta-1) c_{0} n_{\mathrm{Cd}, 0} t}}{\beta-e^{-k_{2}(\beta-1) c_{0} n_{\mathrm{Cd}, 0} t}}\right)
$$

Here, $n_{\mathrm{Cd}, 0}$ is the amount of the $\mathrm{Cd}$ precursor in the reaction mixture, $\beta$ is the molar ratio of the selenium and cadmium precursor, $k_{2}$ is the second order rate constant of the monomer formation reaction and $c_{0}$ is the inverse of the reaction volume of $12 \mathrm{~mL}$. For reactions at 230 ${ }^{\circ} \mathrm{C}$, the rate constant $k_{2}$ was determined at $0.14 \mathrm{~L} \mathrm{~mol}^{-1} \mathrm{~s}^{-1} \cdot{ }^{13}$

Data analysis - nanocrystal concentration. To estimate the moment the NC concentration reaches $98 \%$ of its final value, we interpolated the NC concentration as obtained for the different aliquots by means of a single exponential buildup:

$$
n_{\mathrm{NC}}(t)=n_{\mathrm{NC}, \infty}\left(1-e^{-k t}\right)
$$

This expression can be obtained by considering the monomer mass balance. Under conditions where monomer generation is rate determining, the number of monomers used for nucleation should equal the difference between the monomer generation rate and the monomer consumption by growth. Hence: 


$$
\frac{V_{c}}{V_{m}} \frac{d n_{N C}}{d t}=G_{M}-K n_{N C}
$$

Here, $V_{c}$ is the volume of a critical nucleus, $V_{m}$ the molar volume of the material precipitating, $G_{M}$ is the monomer generation rate and $K$ is the rate at which monomers adsorb at a single nanocrystal. Under conditions where $V_{c}, G_{M}$ and $K$ are constant, the exponential buildup (Eq S3.3) we used for interpolation purposes is obtained. Interestingly, this exponential buildup provides a satisfactory fit to the time development of the nanocrystal concentration we obtained from the SAXS analysis (see Figure S3.1). Considering the assumptions under which the exponential buildup was derived, a constant $V_{c}$ and $G_{M}$ do not seem problematic in the beginning of the reaction. The assumption of a constant rate constant $k$, on the other hand, is less obvious. Probably, the correspondence between the data and the exponential buildup results from an average over all existing nanocrystals. Even so, the correspondence

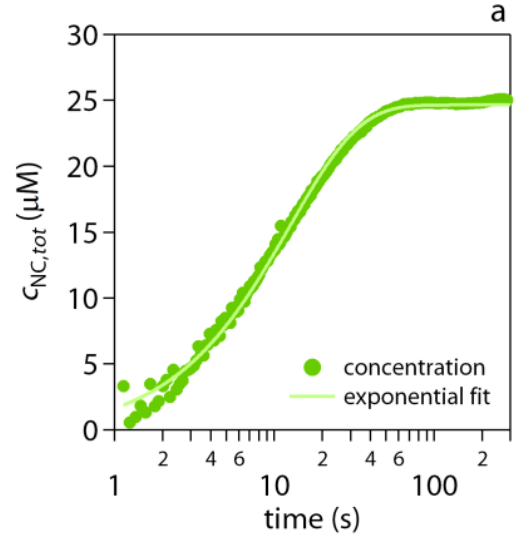

Figure S3.1 | (bottom) Best fit of an exponential buildup to the timedependent nanocrystal concentration as measured by SAXS (see Figure 1 of the manuscript). between the in-situ SAXS and the simple exponential buildup warrants the use of Eq S3.4 to determine the moment at which a synthesis attains $98 \%$ of its final concentration by data interpolation.

\section{S.3.2 PbS nanocrystal synthesis}

Synthesis method. PbS NCs were synthesized according to a previously published procedure with slight modifications. ${ }^{15}$ Lead oleate $(1.2 \mathrm{mmol})$ and $\mathrm{n}$-dodecane $(17 \mathrm{~mL})$ were stirred under a nitrogen flow for $30 \mathrm{~min}$ at room temperature and $30 \mathrm{~min}$ at $120^{\circ} \mathrm{C}$. In a nitrogen-filled glove box, N-dodecyl-N'-phenylthiourea $(0.8 \mathrm{mmol})$ was dissolved in 1-methoxy-2-(2methoxyethoxy)ethane (1.5 mL), taken outside and quickly injected into the lead oleate solution at $120{ }^{\circ} \mathrm{C}$. Aliquots were removed and purified 3 times by aid of toluene/acetone.

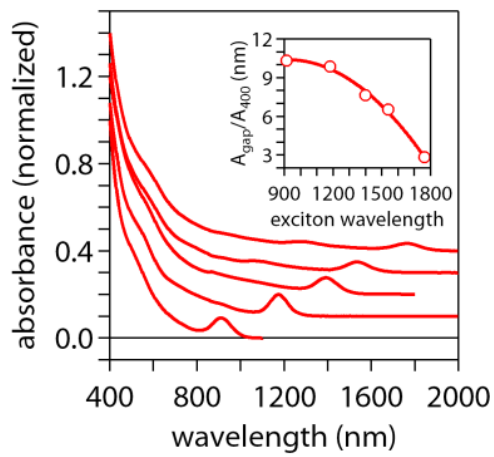

Figure S3.1. $\mathrm{PbS}$ reference spectra and (inset) calibration curve linking the wavelength integrated band-edge absorbance $A_{g a p, \lambda}$ to the absorbance $A_{400}$ at $400 \mathrm{~nm}$. The calibration curve was determined as: $A_{g a p, \lambda} / A_{400}=1.14+0.0200 / \lambda+1.0810^{-5} / \lambda^{2}$ with $\lambda$ in $\mathrm{nm}$.

Data analysis. The NC radius was determined from the peak wavelength of the exaction absorbance using a published, SAXS-based calibration curve. ${ }^{2}$ Since the synthesis produces a 
collection of small clusters in the early stage of the reaction, ${ }^{2}$ we used the integrated band-edge absorbance of quantitative aliquots to obtain the amount $n_{\mathrm{PbS}}$ of $\mathrm{PbS}$ in the reaction mixture. For this purpose, a calibration curve relating the wavelength integrated absorbance $A_{g a p, \lambda}$ of the band-edge transition to the absorbance $A_{400}$ was established by means of reference spectra of purified $\mathrm{PbS}$ dispersions (see Figure S3.1). This calibration curve enabled us to determine the corresponding absorbance at $400 \mathrm{~nm}$ of the nanocrystal in each aliquot from the integrated band-edge absorbance. The amount of $\mathrm{PbS}$ was then obtained using published absorption coefficients at $400 \mathrm{~nm} .{ }^{16}$ Finally, the nanocrystal concentration was obtained by combining the nanocrystal radius and $n_{\mathrm{Pbs}}$. The amount of $\mathrm{PbS}$ and the nanocrystal concentration were fitted to Eq S3.2, including an additional $5 \mathrm{~s}$ time delay to account for the delayed nanocrystal formation.


Figure S3.2. (a) Absorbance spectra of reaction aliquot taken as indicated after the start of a $\mathrm{PbS}$ synthesis based on reacting lead oleate with N-dodecyl-N'-phenylthiourea. (b) Nanocrystal radius as obtained from SAXS analysis. (c) Amount of (blue) PbS and (red) nanocrystals as obtained from the SAXS analysis. Full lines are fits to Eq S3.2. We took the points where both fits attain $98 \%$ of their final value to identify the end of the nucleation and the growth to full yield period.

Reaction development. Figure S3.2 shows the absorbance spectra, the corresponding radii and the amount of $\mathrm{PbS}$ and the $\mathrm{NC}$ concentration as measured for the different aliquots taken at the time indicated. After $5 \mathrm{~s}$, only a small fraction of NCs is formed; a pronounced exciton feature and a sizeable NC concentration are only observed after $15 \mathrm{~s}$. Using the estimated time the nanocrystal concentration (68 s) and the yield (164 s) attain $98 \%$ of their maximal value, we estimate that nucleation last during $\sim 40 \%$ of the synthesis, a number that is even larger than what we found for the CdSe syntheses shown in the main text.

\section{S.3.3 CdS nanocrystal synthesis}

Precursor synthesis. Cadmium oleate (cadmium to oleic acid ratio 1:3) was prepared by mixing $\mathrm{CdO}$ and oleic acid in a 1:3 molar ratio, degassing for $1 \mathrm{~h}$ at $100{ }^{\circ} \mathrm{C}$ under a nitrogen flow, and dissolving the cadmium oxide under a nitrogen atmosphere between 250 and $300{ }^{\circ} \mathrm{C}$ until the mixture became clear. TOP-S solutions (solutions of tri-octylphosphine sulfide in TOP) were prepared by dissolution of elemental sulfur in TOP at room temperature under a nitrogen atmosphere.

Nanocrystal synthesis. The synthesis of CdS nanocrystals was carried out as described in literature. ${ }^{13}$ A mixture of cadmium oleate $(0.4 \mathrm{mmol})$, hexadecylamine $(1.6 \mathrm{mmol})$, oleic acid 
$(0.2 \mathrm{mmol})$ and ODE $(9.46 \mathrm{~mL})$ was stirred under a nitrogen flow for $30 \mathrm{~min}$ at room temperature and $60 \mathrm{~min}$ at $100{ }^{\circ} \mathrm{C}$. The nitrogen flow was stopped, and still under nitrogen, the temperature was raised to $280{ }^{\circ} \mathrm{C}$ and $2 \mathrm{~mL}$ of a $1 \mathrm{M}$ TOP-S solution $(2 \mathrm{mmol})$ were injected, and the reaction was performed at $260{ }^{\circ} \mathrm{C}$. Aliquots were removed and purified 3 times by addition of toluene, isopropanol and methanol, all in a 1:1:1 ratio relative to the volume of the reaction mixture.

Data analysis. The amount of CdS, the nanocrystal radius, and the amount of NCs were all obtained from the analysis of the absorbance of quantitative reaction aliquots. To determine the amount of CdS, we used an intrinsic absorption coefficient $\mu_{i}=2.0110^{5} \mathrm{~cm}^{-1}$ at $300 \mathrm{~nm}$, as deduced from published optical constants for bulk CdS. ${ }^{17}$ The NC radius was determined from the peak wavelength of the exciton absorbance using a published, SAXS-based calibration curve. ${ }^{2}$ The nanocrystal concentration was calculated from the combination of the amount of $\mathrm{CdS}$ and the nanocrystal radius. The buildup of the amount of $\mathrm{CdS}$ and the amount of nanocrystals were fitted to Eq S3.1 and EqS3.2, respectively.
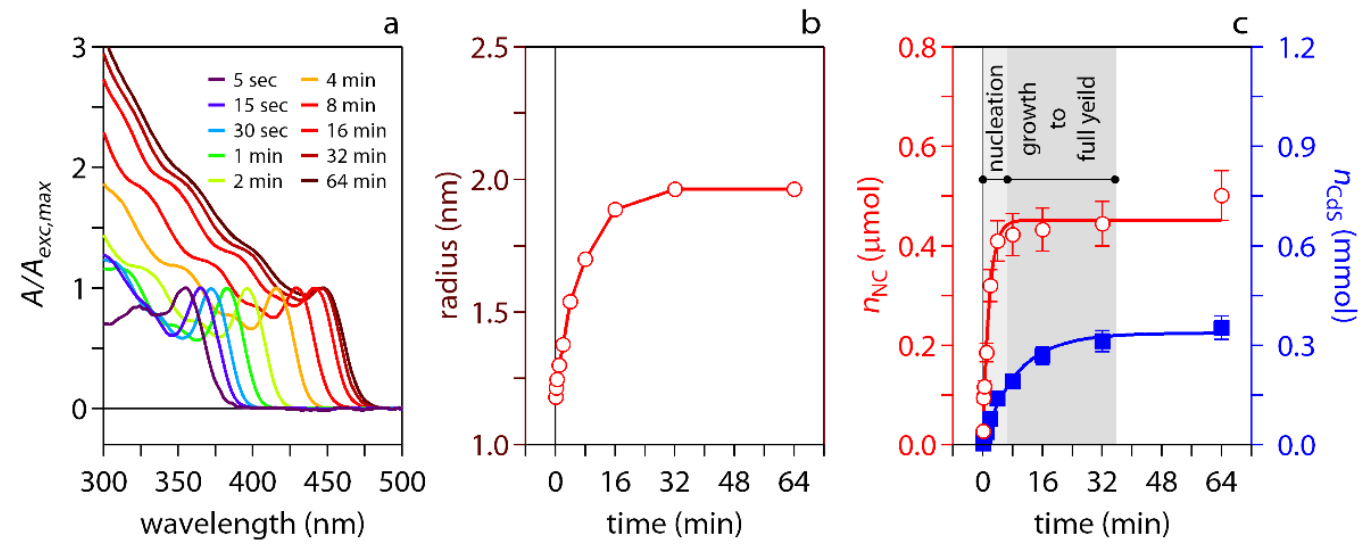

Figure S3.3. (a) Absorbance spectra of reaction aliquot taken as indicated after the start of a $\mathrm{CdS}$ synthesis based on reacting cadmium oleate with tri-octylphosphine sulfur. (b) Nanocrystal radius estimated from the spectral position of the CdSe NC band-edge transition. (c) Amount of (blue) CdS and (red) nanocrystals as obtained from the absorbance at $300 \mathrm{~nm}$ of quantitative reaction aliquots in combination with the nanocrystal radius. Full lines are fits to Eq S3.1 and S3.2, respectively. We took the points where both fits attain 98\% of their final value to identify the end of the nucleation and the growth to full yield period.

Reaction development. Figure S3.3 shows the absorbance spectra, the corresponding radii and the amount of $\mathrm{CdS}$ and the NC concentration as measured for the different aliquots taken at the time indicated. Using the estimated time the nanocrystal concentration $(6.5 \mathrm{~min})$ and the yield (35.4 min) attain $98 \%$ of their maximal value, we estimate that nucleation last during $~ 18 \%$ of the synthesis, a number in line with the CdSe syntheses shown in the main text. 


\section{S.4 Nanocrystal growth}

\section{S.4.I The nanocrystal growth rate}

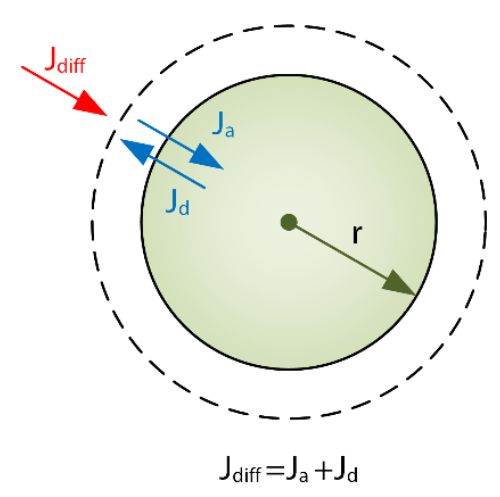

Figure S4.1. Representation of the different fluxes involved in nanocrystal growth. The dashed line represents the surface at which boundary condition S4.5 is applied, which was artificially displaced from the true nanocrystal surface to highlight the interrelation between the fluxes.

We define the NC growth rate $j_{G}$ as the rate of change of the nanocrystal radius $r$ :

$$
j_{G}=\frac{d r}{d t}
$$

To obtain an expression for $j_{G}$ as a function of the NC radius, we assume that growth involves the diffusion of monomers to the $\mathrm{NC}$ surface, and the adsorption or desorption of monomers at the NC surface, see Figure S4.1. Considering spherical NC, the concomitant fluxes $J_{d i f f}, J_{a}$ and $J_{d}[\mathrm{~mol} / \mathrm{s}]$ can be written as:

$$
\begin{aligned}
& J_{\text {diff }}=4 \pi r D([\mathrm{M}]-[\mathrm{M}(r)]) \\
& J_{a}=4 \pi r^{2} k_{a}(r)[\mathrm{M}(r)] \\
& J_{d}=-4 \pi r^{2} k_{d}(r)
\end{aligned}
$$

Here, $D$ is the monomer diffusion coefficient, $[\mathrm{M}]$ and $[\mathrm{M}(r)]$ are the monomer concentration in the reaction mixture and at the surface of the nanocrystal, respectively. The respective symbols $k_{a}(r)$ and $k_{d}(r)$ denote the rate constants for monomer adsorption and desorption, which are explicitly seen as radius dependent quantities. Note that in writing Eq S4.4, we assumed that the diffusion layer thickness strongly exceeds the NC radius.

At the NC surface, the different fluxes are related by a boundary condition:

$$
J_{\text {diff }}=J_{a}+J_{d}
$$

Accordingly, we obtain $[\mathrm{M}(r)]$ as:

$$
[\mathrm{M}(r)]=\frac{D[\mathrm{M}]+r k_{d}(r)}{D+r k_{a}(r)}
$$


Hence, the net monomer flux $J$ towards the NC surface can be written as:

$$
J=J_{a}+J_{d}=4 \pi r^{2} D \frac{k_{a}(r)[\mathrm{M}]-k_{d}(r)}{D+r k_{a}(r)}=4 \pi r^{2} D[\mathrm{M}]_{0} \frac{S-\frac{k_{d}(r)}{k_{a}(r)[\mathrm{M}]_{0}}}{\frac{D}{k_{a}(r)}+r}
$$

In the latter equation, we have introduced the monomer solubility $[\mathrm{M}]_{0}$ and the supersaturation $S$ as the ratio $[\mathrm{M}] /[\mathrm{M}]_{0}$ between the actual monomer concentration and the solubility. From Eq $\mathrm{S} 4.7$, the growth rate is eventually obtained as:

$$
j_{G}=\frac{V_{m}}{4 \pi r^{2}} J=D V_{m}[\mathrm{M}]_{0} \frac{S-e^{\frac{2 \gamma V_{m}}{r R T}}}{\frac{D}{k_{a}(r)}+r}
$$

Here, we have introduced the relation between the adsorption and desorption rate constants that follows from equilibrium considerations, where $\gamma$ is the surface tension, $V_{m}$ the molar volume of the condensed phase, $R$ the gas constant and $T$ the absolute temperature. Indeed, when the monomer concentration is equal to the nanocrystal solubility $[\mathrm{M}]_{0, r}$, the adsorption and desorption flux should balance. Hence, using the Kelvin equation, we have:

$$
\frac{k_{d}(r)}{k_{a}(r)}=[\mathrm{M}]_{0, r}=[\mathrm{M}]_{0} e^{\frac{2 \gamma V_{m}}{r R T}}
$$

\section{S.4.2 Rate constants based on a linear free-energy argument}

In order to use the expression $\mathrm{S} 4.8$ for the growth rate, an explicit expression of the radiusdependence of $k_{a}(r)$ is needed. Here, a possible answer follows from extending the Kelvin equation with a linear free energy argument. The argument goes as follows. As indicated in Eq S4.9, the solubility of small nanocrystals increases with decreasing radius. This can be interpreted as an increase of the chemical potential of the monomers in a nanocrystal solid as compared to a bulk solid. As outlined in Figure S4.2a, a linear free energy argument states that this increase of the free energy of the final state induces a proportional increase of the activation energy of the forward reaction, i.e., the adsorption reaction. We thus have:

$$
k_{a}(r)=k_{a}(\infty) e^{-\alpha \frac{2 \gamma V_{m}}{r R T}}
$$

Using this relation, we obtain the expression of the growth rate as proposed by Talapin et al., which predicts a gradual increase of $k_{a}(r)$ with increasing radius that levels of at $k_{a}(\infty)$ in the bulk limit. ${ }^{18}$ Note that by introducing the critical radius $r_{c}=2 \gamma V_{m} / R T \ln S$, Eqs S4.8, S4.9 and $\mathrm{S} 4.10$ can be combined to write the growth rate as:

$$
j_{G}=D V_{m}[\mathrm{M}]_{0} \frac{S-S^{\frac{r_{c}}{r}}}{r+\frac{D}{k_{a}(r)}}=D V_{m}[\mathrm{M}]_{0} \frac{S-S^{\frac{r_{c}}{r}}}{r+\frac{D}{k_{a}^{\infty}} S^{\alpha^{\frac{r_{c}}{r}}}}
$$





Figure S4.2. (a) Representation of bulk and nanocrystal solubility, where the enhanced solubility of the nanocrystals is related to an increased chemical potential of the monomers incorporated in nanocrystals as compared to bulk. Through a linear free energy argument, the activation energy for monomer adsorption changes proportionally with this increase of the chemical potential, ${ }^{18}$ as described by the transfer coefficient $\alpha$. (b) Growth rate obtained in a reaction-limited regime using an adsorption rate constant as described by a linear free energy relationship.

Combined with Eq 4.8, Eq S4.10 yields a growth rate that increases with increasing radius as shown in Figure S4.2b and Figure 3c of the main text. Here, the calculation was done using the parameter setting as summarized in Table S4.1.

Table S4.1. Parameter setting for the calculation of the growth rate under diffusion and reaction control as shown in Figure 3b-c of the main text and Figure S4.2b.

\begin{tabular}{|c|l|l|l|}
\hline Symbol & Parameter Description & Value & Unit \\
\hline$D$ & Monomer diffusion coefficient & $5 \mathrm{e}-10$ & $\mathrm{~m}^{2} / \mathrm{s}$ \\
\hline$v_{0}$ & Volume per CdSe unit cell & $5.59 \mathrm{e}-29$ & $\mathrm{~m}^{3}$ \\
\hline$[\mathrm{M}]_{0}$ & Monomer solubility & $1 \mathrm{e}-7$ & $\mathrm{~mol} / \mathrm{m}^{3}$ \\
\hline$k_{\mathrm{a}}(\infty)$ & Rate constant for monomer adsorption, bulk limit & $4 \mathrm{e}-3$ & $\mathrm{~m} / \mathrm{s}$ \\
\hline$\gamma$ & Surface tension & 0.2 & $\mathrm{~J} / \mathrm{m}^{2}$ \\
\hline$S$ & Supersaturation & 50 & \\
\hline
\end{tabular}

\section{S.4.3 Growth of a bimodal size distribution}

We write the growth rate under conditions that the desorption reaction is negligible in a generic way as: ${ }^{19}$

$$
j_{G}(r)=\frac{A}{r^{n}} S
$$

Here, $A$ is a rate constant and $S$ is the supersaturation. In Eq (S4.12) is a convenient way of expressing the growth rate, since the situation $n=0$ would correspond to reaction limited growth in the limit of large nanocrystals, whereas $n=1$ would yield diffusion limited growth under the same conditions. Since $j_{G}(r)=d r / d t$, we have: 


$$
r^{n} d r=A S(t) d t
$$

Integration left and right thus yields:

$$
\frac{1}{n+1}\left(r^{n+1}-r_{0}^{n+1}\right)=\int_{0}^{t} A S(t) d t
$$

Since Eq (S4.14) applies to both sets of nanocrystals in a bimodal ensemble, we have:

$$
r_{1}^{n+1}-r_{0,1}^{n+1}=r_{2}^{n+1}-r_{0,2}^{n+1}
$$

So for $n=0$ and $n=1$, we obtain:

$$
\begin{aligned}
& r_{1, \text { diffusion }} \approx \sqrt{r_{2}^{2}+\left(r_{1,0}^{2}-r_{2,0}^{2}\right)} \\
& r_{1, \text { reaction }} \approx r_{2}+\left(r_{1,0}-r_{2,0}\right)
\end{aligned}
$$

Similar expressions for any value of $n$ are readily obtained.

\section{S.4.4 Local approximation of functions as $1 / r^{n}$}

Equation 4 in the main text writes the growth rate in general as:

$$
\frac{d n_{M}}{d t}=\frac{1}{V_{m}} \frac{d V_{N C}}{d t}=4 \pi r^{2}[\mathrm{M}] \frac{k}{r^{n}}
$$

Here, we use this relation as a heuristic model to explore different degrees of superfocusing, as characterized by the exponent $n$. While heuristic, this approach has general relevance since any function $f(r)$ can be locally approximated by means of Eq 4 by an appropriate choice of $n$. To understand this, we start by introducing a local approximation for $f(r)$ as:

$$
f(r)=\frac{A}{r^{n}}
$$

Replacing both sides of the equation by a first order Taylor expansion, we obtain:

$$
f(r)+\frac{d f}{d r} d r=\frac{A}{r^{n}}-n \frac{A}{r^{n+1}} d r
$$

Hence, an expression of the kind $A / r^{n}$ will locally describe $f(r)$ provided that we take the exponent $n$ as:

$$
n=-\frac{r}{f} \frac{d f}{d r}
$$




\section{S.5 Bi-modal size distribution experiments}

\section{S.5.I Experimental method}

A mixture of cadmium oleate $(0.2 \mathrm{mmol})$, hexadecylamine $(0.6 \mathrm{mmol})$, oleic acid $(0.8 \mathrm{mmol})$, and octadecene (total volume $=10 \mathrm{~mL}$ ) was stirred under a nitrogen flow for $1 \mathrm{~h}$ at $120^{\circ} \mathrm{C}$. The nitrogen flow was stopped, and still under nitrogen, the temperature was raised to $\mathrm{X}+15^{\circ} \mathrm{C}$ and $2 \mathrm{~mL}$ of a $1 \mathrm{M}$ TOP-Se solution $(2 \mathrm{mmol})(\mathrm{Cd}: S e \mathrm{1}: 10)$ was injected. After injection, the reaction temperature was stabilized at $\mathrm{X}^{\circ} \mathrm{C}$. After $4 \mathrm{~min}$, an additional $0.4 \mathrm{mmol}$ of $\mathrm{Cd}(\mathrm{OA})_{2}$ was injected and aliquots were taken at different reaction times from $5 \mathrm{~s}$ to $600 \mathrm{~s}$ after this second injection step. The same reaction was carried out at $\mathrm{X}=200,230$, and $270^{\circ} \mathrm{C}$.

Note that this procedure is highly similar to what has been discussed in Supplemental Information S3. In this case, however, oleic acid instead of stearic acid was used as the carboxylic acid and the molar excess of the selenium precursor was increased to 10:1, such that the second injection of cadmium oleate can effectively start a highly similar nucleation event as the first injection.

\section{S.5.2 Reaction development without additional injection}
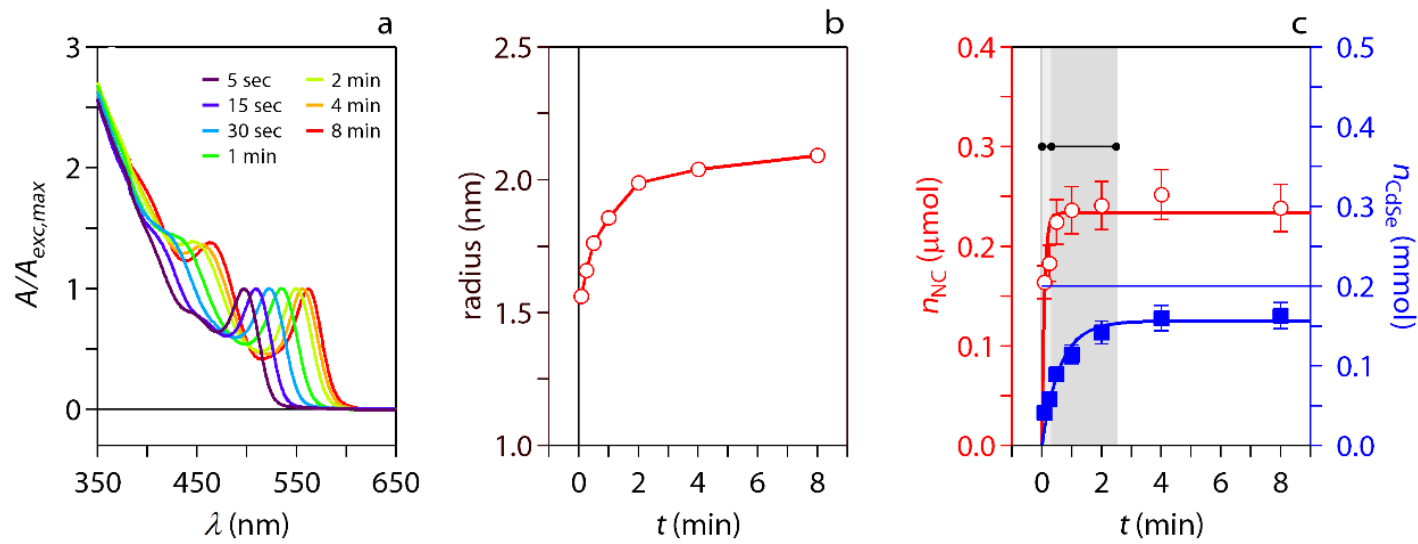

Figure S5.1. (a) Normalized absorbance of aliquots taken at the indicated time from the CdSe reaction mixture. (b) Development of the nanocrystal radius as estimated from the absorbance spectra shown in panel a using a SAXS-based sizing curve. ${ }^{2}$ (c) Development of the amount of (blue) CdSe and (red) nanocrystals as estimated from the absorbance spectra shown in panel a. The light and dark grey areas delineate the period in which the nanocrystal concentration and the CdSe yield reach $98 \%$ of the final value.

Figure S5.1 represents the reaction development for the synthesis as described above, carried out at $245^{\circ} \mathrm{C} / 230^{\circ} \mathrm{C}$ injection/growth temperature. The absorbance spectra give no indication of the formation of a second population. In this case, $98 \%$ of the final yield is reached after 2.5 minutes, while the nanocrystal concentration takes $0.31 \mathrm{~min}$ to reach a constant value. Hence, we estimate that the nucleation period for this reaction lasts for $\sim 12.5 \%$ of the reaction time.

\section{S.5.3 Reaction development after additional injection}

Figure S5.2a represents the absorption spectra of reaction aliquots taken at the indicated time after the second injection of $\mathrm{Cd}(\mathrm{OA})_{2}$ for the reaction carried out at $230^{\circ} \mathrm{C}$ injection/growth temperature. The corresponding photoluminescence spectra have been represented in Figure $4 \mathrm{~b}$. In particular in the spectra recorded until $60 \mathrm{~s}$ after the second injection, the band-edge 
absorption feature of the smaller subset can be identified. However, an accurate determination of the band-edge position from these spectra is difficult, a result that motivated us to analyze the bimodal size distribution by photoluminescence spectroscopy. The thus obtained bandedge positions have been added as markers to the spectra shown in S5.2a.


Figure S5.2. (a-b) Absorbance spectra of aliquots for the bimodal distribution experiment at $230{ }^{\circ} \mathrm{C}$, corresponding to Figure $4 \mathrm{~b}$ in the main text. The spectra are recorded on quantitative aliquots taken at the indicated moment after the second injection of $\mathrm{Cd}(\mathrm{OA})_{2}$. (b) (markers) Amount of CdSe obtained from the absorbance spectra shown in (a) and (full line) fit of these data to Eq. S3.2 from which we obtain a second order rate constant $k_{2}=0.125 \pm$ $0.015 \mathrm{~L} /(\mathrm{mol} \cdot \mathrm{s})$.

Through the absorption of each quantitative aliquot, we could also determine the additional amount of CdSe formed by the second injection. As shown in Figure S.5.2b one sees that the second injection leads to a conversion yield of $70 \%$, slightly smaller than typically found for a single injection reaction. Moreover, a fit to Eq S.3.2 yields a second order rate constant $k_{2}=$ $0.125 \pm 0.015 \mathrm{~L} /(\mathrm{mol} \cdot \mathrm{s})$. This rate constant coincides within the measurement error with the published value of $k_{2}=0.14 \pm 0.01 \mathrm{~L} /(\mathrm{mol} \cdot \mathrm{s}) .{ }^{13}$ Given these observations, we conclude that the CdSe formation is indeed independent of the presence of nanocrystals.

\section{S.5.4 Luminescence calibration}


Figure S5.3. (a-b) Absorbance spectra of aliquots for the CdSe synthesis used to determine the exciton peak absorbance wavelength $v s$. peak emission wavelength calibration curve. The 
same color is used to represent absorbance and emission from the same aliquot. (c) Resulting calibration curve, including an expression for the best fitting linear trendline.

To establish a calibration curve linking the wavelength of maximum exciton emission to the wavelength of maximum exciton absorbance, we used two CdSe synthesis as shown in Figure S5.1, where we changed the oleic acid:cadmium oleate ratio to have a sufficiently wide span of the exciton peak absorbance and emission. As shown in Figure S5.3, by taking different aliquots during such a synthesis, a relation between the wavelength where the exciton absorbance and the emission peak can be established. A linear fit to such datapoints yields a calibration curve that we used to turn the emission wavelength in an absorbance wavelength. The latter was recalculated as a nanocrystal radius using a published, SAXS-based calibration curve. ${ }^{2}$

\section{S.5.5 Superfocusing analysed by UV-Vis absorption spectroscopy}

As shown in Figure S5.2a, the bimodal distribution created by the second injection of $\mathrm{Cd}(\mathrm{OA})_{2}$ is difficult to quantify by means of UV-Vis absorption spectra for the synthesis as described in S5.1, hence our use of photoluminescence spectroscopy to determine the average radius of both sets of nanocrystals in Figure 4. While band-edges obtained from photoluminescence correspond to the few identifiable features of both sets of nanocrystals in the absorption spectra shown in Figure S5.2a, a photoluminescence analysis may create artifacts since only emissive nanocrystals are measured.

To avoid such issues, we extended the study on bimodal size distributions and superfocusing to a different $\mathrm{CdSe}$ synthesis, first described by Flamee et al. Here, $\mathrm{CdO}(0.4 \mathrm{mmol})$ was added to $10 \mathrm{~mL}$ of ODE together with $1.2 \mathrm{mmol}$ of oleic acid in a three neck flask with cooler under air. The mixture was heated up to $270{ }^{\circ} \mathrm{C}$ to dissolve the red $\mathrm{CdO}$ in ODE by the formation of a cadmium carboxylate complex. The formation of CdSe nanocrystals was initiated by injecting a $2 \mathrm{~mL}$ solution of ODE containing $0.05 \mathrm{mmol}$ of black Se powder. The growth temperature was set at $260{ }^{\circ} \mathrm{C}$. After $20 \mathrm{~s}$ of reaction, a similar injection of $0.05 \mathrm{mmol}$ followed, while keeping the reaction temperature at $260{ }^{\circ} \mathrm{C}$, and aliquots were taken at regular intervals.

As can be seen in Figure S5.4, the second injection creates in this case a clearly identifiable absorption feature of the smaller subset that stands out relative to the shorter wavelength absorption features of the larger subset. Identifying the average radius of the initial and the newly formed subset from these absorption spectra, we again find that the smaller subset grows significantly faster than the larger set than predicted based on the $1 / r$ dependence of diffusionlimited growth, i.e., superfocusing.
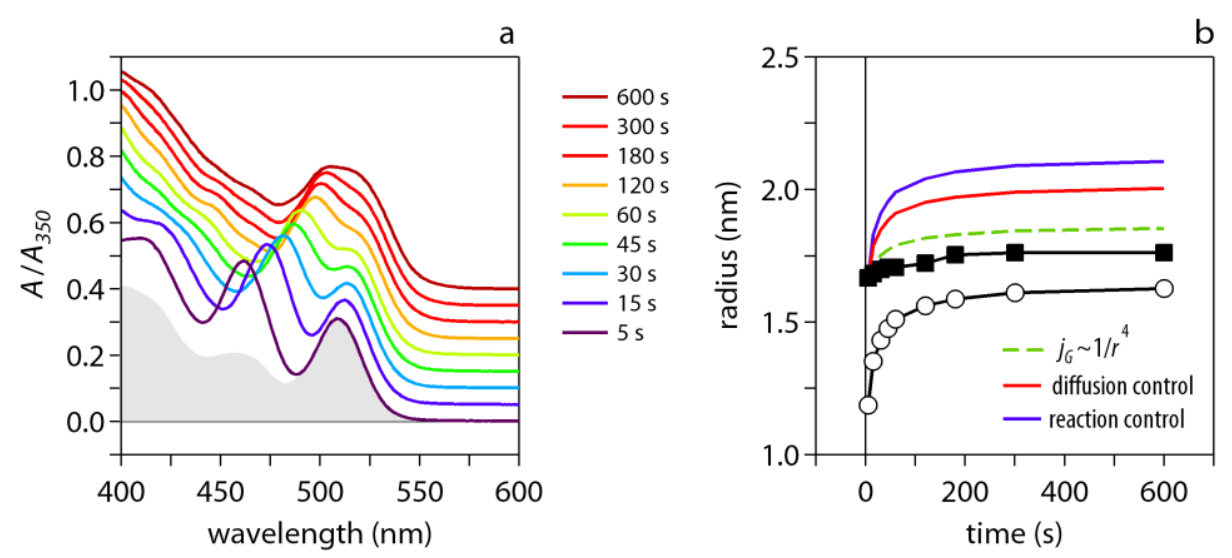
Figure S5.4. Observation of superfocusing in a CdSe synthesis based on the reaction between cadmium oleate and black selenium. (a) (colored lines) Absorbance spectra recorded at the indicated times after a second injection of black selenium and (filled gray trace) reference spectrum of a CdSe nanocrystal batch obtained with the same synthesis after a single precursor injection. Given this reference spectrum, we attribute the two absorption features at $\sim 510 \mathrm{~nm}$ and $\sim 460 \mathrm{~nm}$ in the UV-Vis spectrum obtained $5 \mathrm{~s}$ after the second precursor injection to the initial and newly formed set of nanocrystals, respectively. (b) Variation of the nanocrystal diameter as a function of time for the (black squares) initial set and (open circle) newly formed set of nanocrystals in the reaction mixture. The full blue and full red line indicate the expected evolution of the diameter of the large set under diffusion and reaction control. The green dashed line represents the evolution expected for a growth exponent $n=4$. 


\section{S.6 Kinetic reaction modeling}

\section{S.6.I The reaction chemistry of CdSe formation}

According to literature, the formation of $\mathrm{CdSe}$ from a cadmium carboxylate and trioctylphosphine selenium takes place by means of a two-step mechanism in which both precursors first react to form a compound typically referred to as the monomer. ${ }^{20-21}$ This monomer is the actual solute or precipitant. The following chemical reactions equations for the precursor conversion have been proposed: $:^{20-21}$

$$
\mathrm{TOPSe}+\mathrm{Cd}(\mathrm{RCOO})_{2} \rightleftharpoons(\mathrm{TOP}) \mathrm{Se}-\mathrm{Cd}(\mathrm{RCOO})_{2} \rightarrow \mathrm{CdSe}+\mathrm{TOPO}+\mathrm{RCOOOCR}
$$

Here, the CdSe formula unit represents the CdSe monomer in the reaction mixture. Moreover, it was found that the byproducts of the precursor conversion - in particular TOPO - are produced at the same rate as the solid CdSe is formed. Therefore, several authors proposed that the conversion reaction from precursors to monomers determines the overall rate at which solid material is formed through nucleation and growth. ${ }^{13,21}$ This conclusion was confirmed in the case of $\mathrm{CdS}$ formation by the finding that the formation rate of $\mathrm{CdS}$ was independent of the presence of seed nanocrystals in the reaction mixture or not. ${ }^{22}$ Building on this insight, Abe et al. analyzed the CdSe formation rate as a function of the concentration of the $\mathrm{Cd}(\mathrm{RCOO})_{2}$ and TOPSe in the reaction mixture and showed that the formation rate follows a simple rate law that is first order in the concentration of either precursor. ${ }^{13}$ Importantly, the buildup of CdSe after the second injection in the bimodal size distribution experiments yields the same second order rate constant as published previously by Abe et al.. This observation confirms that the formation of $\mathrm{CdSe}$ is determined by the monomer formation rate, and is independent of the presence or not of CdSe nanocrystals

\section{S.6.2 Modeling colloidal crystallization}

In the literature, two main approaches have been developed to describe nucleation and growth in crystallization reactions. ${ }^{23}$ A first alternative - referred to as the population balance equation model in ref ${ }^{23}$ - involves a continuous approach in which the nanocrystal population balance is expressed through a partial differential equation that takes time and the nanocrystal radius as independent variables. A second - dubbed the kinetic rate equation method - translates nucleation, growth and dissolution into a set of discrete ordinary differential equations, each addressing a single, discrete nanocrystal size. For computational convenience, both methods can be coupled by using separate rate equations for small nanocrystals, and a continuous approach for larger nanocrystals. In the case of precipitation reactions involving a homogenous monomer formation, the continuous approach has been used to describe nucleation and growth of iron oxide and CdSe nanocrystals, ${ }^{13},{ }^{24}$ while a hybrid methodology was implemented by Rempel et al. ${ }^{25}$ For reactions involving surface-catalyzed growth, extensive modeling of nucleation and growth has been accomplished by the kinetic rate equation method. ${ }^{26-27}$

Comparing both approaches, Vetter et al. concluded that: ${ }^{23}$

the KRE and PBE model deliver similar descriptions of the process and that the dependence of the two models on the key parameters given above is consistent. Nevertheless, the KRE model in general allows obtaining deeper insight into the behavior of both sub-and supercritical crystals. 
This statement aligns with the observation that the continuous simulations of a hot injection synthesis carried out by Abe et al. ${ }^{13}$ and the hybrid simulations proposed by Rempel et al. ${ }^{25}$ yield a comparable development of the concentration distribution as a function of time and particle size. Even so, one should realize that a continuous approach in which nucleation is modelled by means of classical nucleation theory will not give atomistic insight in the nucleation process.

\section{S.6.3 The population balance equations}

All simulations are based on the model equations reported and explained previously. ${ }^{13}$ Based on the literature insight in the CdSe synthesis, the modelling approach is based on a kinetic scheme where injected precursors $\mathrm{P}$ react to form a solute or monomer $\mathrm{M}$, which precipitates to form nanocrystals either by nucleation or growth, a mechanism we succinctly represent as:

$$
\mathrm{P} \rightarrow \mathrm{M} \rightleftharpoons \mathrm{NC}
$$

While the formation of a compound such as CdSe requires multiple precursors, we will only consider a single precursor species for the reaction simulations. This approach reflects the finding that the CdSe synthesis can be carried out under pseudo first-order conditions, for example by using a substantial excess of the selenium precursor.

Following scheme 6.2, the central quantities in the modeling are (1) the concentration distribution $c(r, t)$ of NCs in $\left[1 / \mathrm{m}^{4}\right]$ - where the product $c(r, t) d r$ yields the concentration of NCs with a radius between $r$ and $r+d r$ at time $t$, (2) the supersaturation $S(t)$, which is defined as the ratio between the actual concentration of the solute or monomer and its equilibrium concentration $[\mathrm{M}]_{0}$ and (3) the precursor concentration $[\mathrm{P}]$. For simplicity, the model assumes a single precursor.

The concentration distribution $c(r, t)$ changes with time since new NCs nucleate (index $N$ ) and existing NCs grow (index $G$ ):

$$
\frac{\partial c}{\partial t}=\left.\frac{\partial c}{\partial t}\right|_{N}+\left.\frac{\partial c}{\partial t}\right|_{G}=J_{N} \delta\left(r-r_{C}\right)-\frac{\partial\left(j_{G} c\right)}{\partial r}
$$

In the above expression, we describe nucleation as the formation of NCs with the critical radius $r_{C}[\mathrm{~m}]$ at a rate $J_{N}\left[1 / \mathrm{m}^{3} \cdot \mathrm{s}\right]$, both given by classical nucleation theory:

$$
\begin{aligned}
& r_{C}=\frac{2 \gamma V_{m}}{R T \ln S} \\
& J_{N}=\frac{2 D}{v_{0}^{5 / 3}} \exp \left(-\frac{16 \pi \gamma^{3} V_{m}^{2} N_{A}}{3(R T)^{3}(\ln S)^{2}}\right)
\end{aligned}
$$

Here, $v_{0}$ is the monomer volume and $N_{A}$ is Avogadro's constant. The growth term in Eq (S6.2) is written in terms of the NC growth rate $j_{G}$, see Eq. S4.8.

Regarding the supersaturation $\mathrm{S}$, we assume that its time-dependence is governed by the generation of monomers from the precursors $G_{M}\left[1 /\left(\mathrm{m}^{3} \cdot \mathrm{s}\right)\right]$ and by their consumption through the nucleation and the growth of nuclei. $d S / d t$ thus reads: 


$$
\frac{d S}{d t}=\frac{1}{[M]_{0}}\left\{G_{M}-\frac{4 \pi r_{C}^{3}}{3 v_{0}} J_{N}+\int_{0}^{\infty} \frac{4 \pi r^{3}}{3 v_{0}} \frac{\partial\left(j_{G} c\right)}{\partial r} d r\right\}
$$

Finally, the monomer generation rate $G_{M}$ is determined by the reaction of the precursor, and therefore the change of the precursor concentration $[\mathrm{P}]$ with time. For simplicity, we assume a first order rate equation:

$$
\frac{d[P]}{d t}=-G_{M}=-k_{1}[P]
$$

\section{S.6.4 Growth-only reaction development}

Mathematical analysis. Following Eq S6.5, the supersaturation changes in the absence of nucleation according to:

$$
\frac{d S}{d t}=\frac{1}{[M]_{0}}\left\{G_{M}+\int_{0}^{\infty} \frac{4 \pi r^{3}}{3 v_{0}} \frac{\partial\left(j_{G} c\right)}{\partial r} d r\right\}
$$

The second part of the right hand side can be rewritten using integration by parts:

$$
\int_{0}^{\infty} \frac{4 \pi r^{3}}{3 v_{0}} \frac{\partial\left(j_{G} c\right)}{\partial r} d r=\left.\left(\frac{4 \pi r^{3}}{3 v_{0}} j_{G} c\right)\right|_{0} ^{\infty}-\int_{0}^{\infty} \frac{4 \pi r^{2}}{v_{0}} j_{G} c d r
$$

To evaluate the first term on the right hand side, we express the total number of monomers $n_{\mathrm{M}}$ incorporated in the nanocrystals as:

$$
n_{\mathrm{M}}=\int_{0}^{\infty} \frac{4 \pi r^{3}}{3 v_{0}} c(r) d r
$$

To have a finite integral, the integrandum should drop to zero for $r \rightarrow \infty$ faster than $1 / r$, hence $c(r)$ must be $\mathrm{O}\left(1 / r^{4}\right)$. Hence, even for $j_{G} \mathrm{O}(1)$, the first term on the right hand side of Eq S6.8 will always be zero.

To evaluate the second term in Eq S6.8 in view of the growth exponent, we write the growth rate as:

$$
j_{G}=\frac{d r}{d t}=V_{m}[\mathrm{M}]_{0} S \frac{k_{a}}{r^{n}}
$$

We obtain accordingly:

$$
\frac{d S}{d t}=\frac{1}{[M]_{0}}\left\{G_{M}-\frac{4 \pi V_{m}[\mathrm{M}]_{0} k_{a}}{v_{0}} S \int_{0}^{\infty} \frac{c(r)}{r^{n-2}} d r\right\}
$$

For $n=2$, the integral in Eq S6.11 yields the total nanocrystal concentration $c_{\text {tot }}$. Since any radius dependence is eliminated, the supersaturation can settle for a time independent value of: 


$$
S=\frac{G_{M} v_{0}}{4 \pi N_{A}[\mathrm{M}]_{0} k_{a} c_{t o t}}
$$

Hence, when $n=2$, growth occurs at constant supersaturation as long as $G_{M}$ is constant. In general, the integral in Eq S6.11 is proportional to the ensemble average $\left\langle 1 / r^{n-2}\right\rangle$. In the case of a quasi-stationary supersaturation, we thus have:

$$
S=\frac{G_{M} v_{0}}{4 \pi N_{A}[\mathrm{M}]_{0} k_{a} c_{t o t}\left\langle 1 / r^{n-2}\right\rangle}
$$

When $n<2,\left\langle 1 / r^{n-2}\right\rangle$ will increase for an ensemble of growing nanocrystals while this average will decrease when $n>2$. As a result, nanocrystals growth reduces the supersaturation when $n<2$, while the supersaturation will increase by nanocrystal growth when $n>2$.

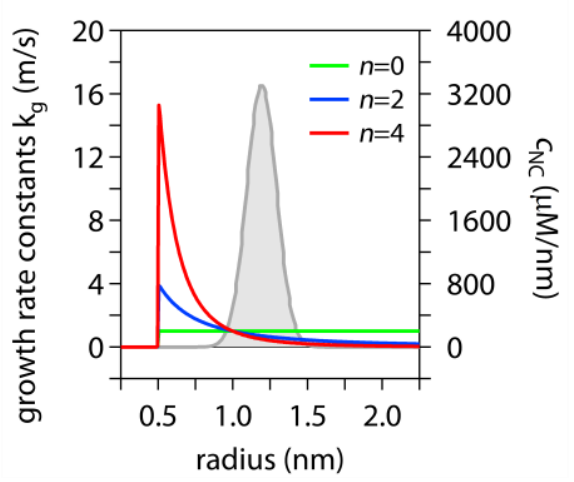

Figure S6.1. (a) (colored) Adsorption rate constants for growth exponents as indicated and (grey) initial concentration distribution used to simulate growth-only reaction development. (b) (colored) Concomitant nanocrystal growth rate, calculated using Eq. S6.14. c. Separation of monomer generation over (blue) nucleation and (green) growth, confirming that monomers are only consumed by growth in this growth-only simulation.

Simulated growth-only development. To illustrate the role of the growth exponent in keeping up the supersaturation and promoting persistent nucleation, we implemented the kinetic reaction simulations for an existing concentration distribution under growth-only conditions. Neglecting monomer diffusion and desorption, we implemented the growth rate as (see Figure S6.1):

$$
j_{G}=\frac{d r}{d t}=V_{m}[\mathrm{M}]_{0} S k_{g}(r)
$$

The adsorption rate constant $k_{g}(r)$ was taken as:

$$
k_{g}(r)=A\left(\frac{r_{c, 0}}{r}\right)^{n} \theta\left(r-r_{0}\right)
$$

Here, $\theta\left(r-r_{0}\right)$ is the Heaviside step function, which we implement with $r_{0}=0.5 \mathrm{~nm}$ to prevent that simulations become unstable at radii close to 0 .

As argued before, growth only reaction development at constant monomer generation rate will concur with a decrease of the supersaturation only when the growth exponent $n<2$. We 
analyzed this point by modeling the growth-only reaction development of an initial concentration distribution corresponding to a Gaussian centered around $r=1.2 \mathrm{~nm}$. Figure S6.2 depicts snapshots of the concentration distribution during this development for $n=0,2,4$. It can be seen that a growth exponent $n=0$ results in a mere displacement of the distribution to larger radii with time, as expected for a radius-independent growth rate, while growth exponents $n=2$ and $n=4$ result in a marked focusing of the size distribution. More interesting is the variation of the supersaturation with time, which indeed drops for $n=0$, stays put for $n=2$ and increases for $n=4$. These simulations thus confirm the general point that focusing with a growth exponent $n>2$ will increase the supersaturation during growth-only reaction development; a mechanism that can prolong nanocrystal nucleation during an actual synthesis.


Figure S6.2. (a-c) Evolution of a nanocrystal concentration distribution under growthonly conditions for different growth exponents as indicated. Size distribution focusing for $n=2$ and $n=4$ is clearly visible. (d) Simulated evolution of the supersaturation for the synthesis shown in panel a-c. (e) Simulated evolution of the average radius and the nanocrystal concentration. (f) Simulated evolution of the standard deviation on the average radius, clearly showing the impact of size focusing.

\section{S.6.5 Synthesis simulations under reaction-limited conditions}

The coupled differential equations S6.2, S6.5 and S6.6 were implemented in COMSOL Multiphysics 5.4. A one-dimensional simulation domain for the $\mathrm{NC}$ radius ranging from 0 to 4 $\mathrm{nm}$ was divided in 502 elements. From 0 to $0.2 \mathrm{~nm}$, an absorbing boundary condition was implemented for computational stability. The nucleation rate $J_{N}$ was implemented as a Gaussian function with a width of $0.02 \mathrm{~nm}$, centered $0.3 \mathrm{~nm}$ above the critical radius. 
Table S6.1. Parameter setting for the kinetic reaction simulations shown in Figure 5 of the main text.

\begin{tabular}{|c|l|l|l|}
\hline Symbol & Parameter Description & Value & Unit \\
\hline$D$ & Monomer diffusion coefficient & $5 \mathrm{e}-10$ & $\mathrm{~m}^{2} / \mathrm{s}$ \\
\hline$v_{0}$ & Volume per CdSe unit cell & $5.59 \mathrm{e}-29$ & $\mathrm{~m}^{3}$ \\
\hline$[\mathrm{M}]_{0}$ & Monomer solubility & $1 \mathrm{e}-7$ & $\mathrm{~mol} / \mathrm{m}^{3}$ \\
\hline$k_{1}$ & First order precursor composition rate constant & $4 \mathrm{e}-3$ & $\mathrm{~m} / \mathrm{s}$ \\
\hline$[\mathrm{P}]_{i}$ & Initial precursor concentration & 27 & $\mathrm{~mol} / \mathrm{m}^{3}$ \\
\hline$\gamma$ & Surface tension & 0.2 & $\mathrm{~J} / \mathrm{m}^{2}$ \\
\hline$A$ & Adsorption rate parameter 1 & $1 / 22$ & $\mathrm{~m} / \mathrm{s}$ \\
\hline$B$ & Adsorption rate parameter 2 & $0.2 \mathrm{e}-9$ & $\mathrm{~m}$ \\
\hline$r_{c, 0}$ & Adsorption rate parameter 3 & $0.8 \mathrm{e}-9$ & $\mathrm{~m}$ \\
\hline
\end{tabular}

Table S6.1 provides on overview of the different values we used for the model parameters (see Eqs S6.2-S6.6). Measurable numbers were used for the monomer volume $v_{0}$, which was taken as the volume of a single spherical CdSe unit in the zincblende CdSe unit cell, the monomer diffusion coefficient $D$, which was chosen according to literature values for free oleic acid ligands obtained from diffusion $\mathrm{NMR},{ }^{28}$ and the initial precursor concentration, which was taken in agreement with the cadmium concentration in the in-situ SAXS experiment. Moreover, the first order rate constant was set in line with the experimental reaction rate, whereas an initial precursor concentration was taken in agreement with the concentration of the limiting reagent in a real hot injection synthesis. Other parameter values, most notable the monomer solubility $[\mathrm{M}]_{0}$, the surface tension $\gamma$, and the adsorption rate parameter $A$ were set so as to obtain simulated concentrations and radii in agreement with experimental reactions. Because of the nature of the reaction, the chemical meaning of these parameter values is limited. Since the monomer concentration is quasi-stationary, monomer consumption by growth depends on the product of the rate constant and the monomer concentration. Hence, while this monomer consumption rate can be measured, its factorization in a rate constant and a monomer concentration requires an independent determination of the monomer concentration. Such measurement, however, has remained elusive in the case of a hot injection synthesis. The result is that reaction simulations with somewhat different combination of parameter settings may yield a synthesis development with a similar outcome in terms of nanocrystal concentration and radius. Note that alternative reaction simulation methods will face similar issues when describing reactions in which precursor conversion is the rate determining step.
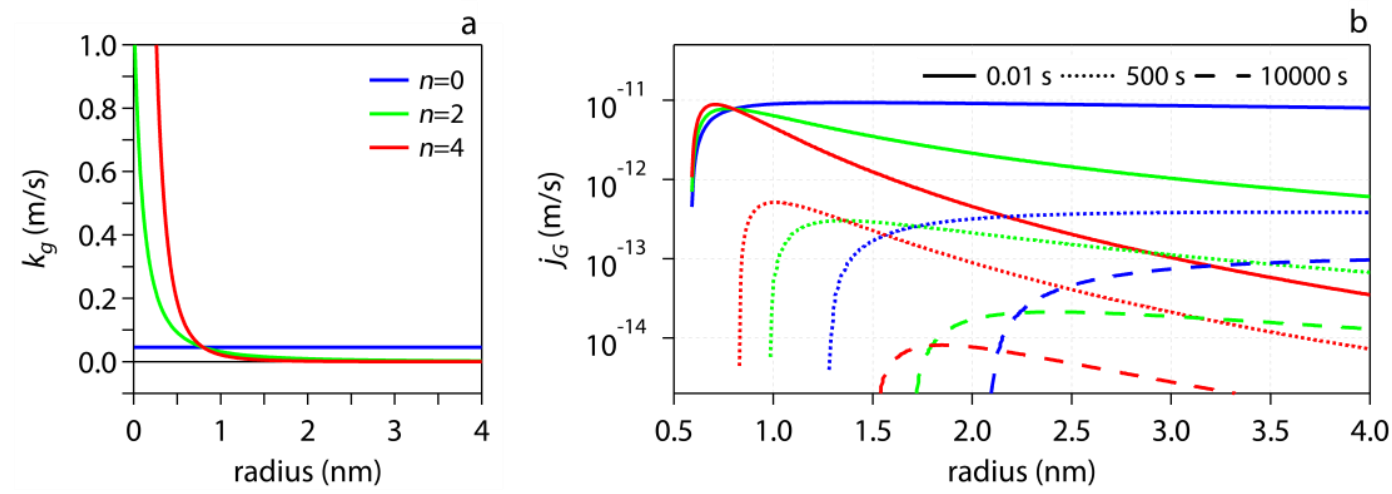

Figure S6.3. (a) Representation of the rate constant of monomer adsorption for different values for the growth exponent as indicated, and $A, B$ and $r_{c, 0}$ parameters as listed in Table S4.2. One sees that the same growth rate is obtained at $r=r_{c, 0}$, regardless of the growth exponent. (b) 
Nanocrystal growth rate linked to the rate constant plotted in panel a at different moments during a reaction simulation. The identical growth rate at $r=r_{c, 0}$ in the initial stage of the reaction and the pronounced difference in focusing as a function of the growth exponent are clearly visible.

To implement superfocusing, the adsorption rate constant $k_{a}(r)$ appearing in Eq. S4.9 was taken as:

$$
k_{a}(r)=A \frac{\left(r_{c, 0}+B\right)^{n}}{(r+B)^{n}}
$$

This expression ensures that regardless of the growth exponent $n$, the same rate constant is obtained at a radius $r=r_{c, 0}$. This point is exemplified in Figure S6.3. In this way, the balancing between nucleation and growth is not changed artificially by a change in initial growth rate for different growth exponents. In addition, the small radial offset $B$ avoids the singularity at $r=0$ that render expressions such as Eq S4.11 difficult to implement numerically, while preserving the role of the growth exponent. As outlined in Table S6.1, we set $B$ fixed to $0.2 \mathrm{~nm}$ and took $r_{c, 0}$ equal to $0.8 \mathrm{~nm}$, in close agreement with the critical radius observed throughout the simulations. Finally, $A$ was chosen such that growth is limited by the surface reaction, not by monomer diffusion, and that in combination with all other parameters, the nanocrystal radius reaches $\sim 2 \mathrm{~nm}$ at the end of the reaction; a number in line with the CdSe synthesis studied through SAXS.

\section{S.6.6 Concentration snapshots}

Concentration snapshots in Figures 5a-c where taken at 3.16, 9.47, 28.34, 49.03, 84.83, 146.78, 253.96, 439.4 and $2275.8 \mathrm{~s}$.

\section{S.6.7 Nanocrystal concentration buildup}

In section S.3.1, we showed that the buildup of the nanocrystal concentration $n_{\mathrm{NC}}(t)$ can be approximated by a single exponential:

$$
n_{\mathrm{NC}}(t)=n_{\mathrm{NC}, \infty}\left(1-e^{-k t}\right)
$$

In addition, we found that the experimental buildup of the nanocrystal concentration from in-situ SAXS measurements fits this expression well. As shown in Figure S6.4, the same conclusion applies to the results of the reaction simulations. For the different choices of the growth exponent $n$, we find that Eq S3.3 yields a reasonable fit for the evolution of the nanocrystal concentration with time. Hence, while the description of nucleation through classical nucleation theory discards the underlying chemistry of the nucleation process, the modeling approach provides a good description of the net effect of nucleation, which is the appearance of growing nancrystals.

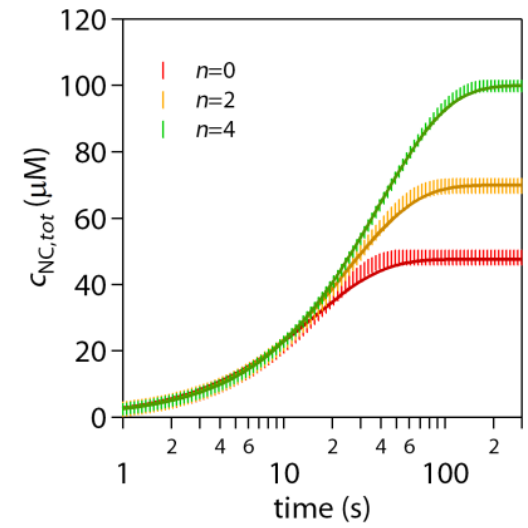

Figure S6.4. (markers) simulated nanocrystal concentration for the different growth exponents as indicated and (lines) best fit to a single exponential buildup. 


\section{References}

1. Qu, L. H.; Peng, Z. A.; Peng, X. G., Alternative routes toward high quality CdSe nanocrystals. Nano Letters 2001, 1 (6), 333-337.

2. $\quad$ Maes, J.; Castro, N.; De Nolf, K.; Walravens, W.; Abécassis, B.; Hens, Z., Size and Concentration Determination of Colloidal Nanocrystals by Small-Angle X-ray Scattering. Chemistry of Materials 2018, 30 (12), 3952-3962.

3. $\quad$ Capek, R. K.; Moreels, I.; Lambert, K.; De Muynck, D.; Zhao, Q.; Van Tomme, A.; Vanhaecke, F.; Hens, Z., Optical Properties of Zincblende Cadmium Selenide Quantum Dots. The Journal of Physical Chemistry C 2010, 114 (14), 6371-6376.

4. Wu, L. H.; Willis, J. J.; McKay, I. S.; Diroll, B. T.; Qin, J.; Cargnello, M.; Tassone, C. J., High-temperature crystallization of nanocrystals into three-dimensional superlattices. Nature 2017, 548 (7666), 197-+.

5. Narayanan, T.; Sztucki, M.; Van Vaerenbergh, P.; Leonardon, J.; Gorini, J.; Claustre, L.; Sever, F.; Morse, J.; Boesecke, P., A multipurpose instrument for time-resolved ultra-smallangle and coherent X-ray scattering. Journal of Applied Crystallography 2018, 51, 1511-1524. 6. Boesecke, P., Reduction of two-dimensional small- and wideangle X-ray scattering data. Journal of Applied Crystallography 2007, 40, S423-S427.

7. Dreiss, C. A.; Jack, K. S.; Parker, A. P., On the absolute calibration of bench-top smallangle X-ray scattering instruments: a comparison of different standard methods. Journal of Applied Crystallography 2006, 39, 32-38.

8. Momma, K.; Izumi, F., VESTA 3 for three-dimensional visualization of crystal, volumetric and morphology data. Journal of Applied Crystallography 2011, 44, 1272-1276.

9. Guinier, A.; Fournet, G., Small-angle scattering of X-rays. John Wiley \& Sons: New York, 1951.

10. Huang, D. H.; Simon, S. L.; McKenna, G. B., Chain length dependence of the thermodynamic properties of linear and cyclic alkanes and polymers. Journal of Chemical Physics 2005, 122 (8).

11. Abecassis, B.; Bouet, C.; Garnero, C.; Constantin, D.; Lequeux, N.; Ithurria, S.; Dubertret, B.; Pauw, B. R.; Pontoni, D., Real-Time in Situ Probing of High-Temperature Quantum Dots Solution Synthesis. Nano Letters 2015, 15 (4), 2620-2626.

12. Sedlak, S. M.; Bruetzel, L. K.; Lipfert, J., Quantitative evaluation of statistical errors in small-angle X-ray scattering measurements. Journal of Applied Crystallography 2017, 50, 621630 .

13. Abe, S.; Capek, R. K.; De Geyter, B.; Hens, Z., Tuning the Postfocused Size of Colloidal Nanocrystals by the Reaction Rate: From Theory to Application. Acs Nano 2012, 6, 42-53.

14. Abe, S.; Capek, R. K.; De Geyter, B.; Hens, Z., Reaction Chemistry/Nanocrystal Property Relations in the Hot Injection Synthesis, the Role of the Solute Solubility. Acs Nano 2013, 7 (2), 943-949.

15. Hendricks, M. P.; Campos, M. P.; Cleveland, G. T.; Jen-La Plante, I.; Owen, J. S., A tunable library of substituted thiourea precursors to metal sulfide nanocrystals. Science 2015, 348 (6240), 1226-1230.

16. Moreels, I.; Lambert, K.; Smeets, D.; De Muynck, D.; Nollet, T.; Martins, J. C.; Vanhaecke, F.; Vantomme, A.; Delerue, C.; Allan, G.; Hens, Z., Size-Dependent Optical Properties of Colloidal PbS Quantum Dots. Acs Nano 2009, 3 (10), 3023-3030.

17. Adachi, S., Optical constants of crystalline and amorphous semiconductors. Kluwer: Norwell, 1999. 
18. Talapin, D. V.; Rogach, A. L.; Haase, M.; Weller, H., Evolution of an Ensemble of Nanoparticles in a Colloidal Solution: Theoretical Study. Journal of Physical Chemistry B 2001, 105, 12278-12285.

19. Dirksen, J. A.; Ring, T. A., Fundamentals of crystallization: Kinetic effects on particle size distributions and morphology. Chemical Engineering Science 1991, 46 (10), 2389-2427.

20. Liu, H. T.; Owen, J. S.; Alivisatos, A. P., Mechanistic study of precursor evolution in colloidal group II-VI semiconductor nanocrystal synthesis. Journal of the American Chemical Society 2007, 129 (2), 305-312.

21. Owen, J. S.; Chan, E. M.; Liu, H.; Alivisatos, A. P., Precursor Conversion Kinetics and the Nucleation of Cadmium Selenide Nanocrystals. Journal of the American Chemical Society 2010, 132 (51), 18206-18213.

22. Nakonechnyi, I.; Sluydts, M.; Justo, Y.; Jasieniak, J.; Hens, Z., Mechanistic Insights in Seeded Growth Synthesis of Colloidal Core/Shell Quantum Dots. Chemistry of Materials 2017, 29 (11), 4719-4727.

23. Vetter, T.; Iggland, M.; Ochsenbein, D. R.; Hänseler, F. S.; Mazzotti, M., Modeling Nucleation, Growth, and Ostwald Ripening in Crystallization Processes: A Comparison between Population Balance and Kinetic Rate Equation. Crystal Growth \& Design 2013, 13 (11), 4890-4905.

24. Kwon, S. G.; Piao, Y.; Park, J.; Angappane, S.; Jo, Y.; Hwang, N.-M.; Park, J.-G.; Hyeon, T., Kinetics of Monodisperse Iron Oxide Nanocrystal Formation by "Heating-Up" Process. J. Am. Chem. Soc. 2007, 129, 12571-12584.

25. Rempel, J. Y.; Bawendi, M. G.; Jensen, K. F., Insights into the Kinetics of Semiconductor Nanocrystal Nucleation and Growth. Journal of the American Chemical Society 2009, 131 (12), 4479-4489.

26. Handwerk, D. R.; Shipman, P. D.; Whitehead, C. B.; Özkar, S.; Finke, R. G., Particle Size Distributions via Mechanism-Enabled Population Balance Modeling. The Journal of Physical Chemistry C 2020, 124 (8), 4852-4880.

27. Handwerk, D. R.; Shipman, P. D.; Whitehead, C. B.; Özkar, S.; Finke, R. G., Mechanism-Enabled Population Balance Modeling of Particle Formation en Route to Particle Average Size and Size Distribution Understanding and Control. Journal of the American Chemical Society 2019, 141 (40), 15827-15839.

28. De Nolf, K.; Capek, R. K.; Abe, S.; Sluydts, M.; Jang, Y. J.; Martins, J. C.; Cottenier, S.; Lifshitz, E.; Hens, Z., Controlling the Size of Hot Injection Made Nanocrystals by Manipulating the Diffusion Coefficient of the Solute. Journal of the American Chemical Society 2015, 137 (7), 2495-2505. 